\begin{tabular}{|c|c|}
\hline Title & Onset of unidirectional pulse propagation in an excitable medium with asymmetric heterogeneity \\
\hline Author(s) & Teramoto, T akashi; Y uan, X iaohui; Bär, Markus; Nishiura, Y asumasa \\
\hline Citation & $\begin{array}{l}\text { Physical Review E, 79(4), } 046205 \\
\text { https://doi.org/10.1103/PhysRevE. } 79.046205\end{array}$ \\
\hline Issue Date & $2009-04$ \\
\hline DOC URL & http:/hdl. handle.net/2115/38556 \\
\hline Rights & @ 2009 A merican Physical Society \\
\hline Type & article \\
\hline File Information & 79-4_046205.pdf \\
\hline
\end{tabular}

Instructions for use 


\title{
Onset of unidirectional pulse propagation in an excitable medium with asymmetric heterogeneity
}

\author{
Takashi Teramoto \\ Faculty of Photonics Science, Chitose Institute of Science and Technology, Chitose 066-8655, Japan \\ Xiaohui Yuan \\ Meme Media Laboratory, Hokkaido University, Sapporo 060-0812, Japan \\ Markus Bär \\ Physikalisch-Technische Bundesanstalt, Abbestrasse 2-12, 10587 Berlin, Germany \\ Yasumasa Nishiura \\ Research Institute for Electronic Science, Hokkaido University, Sapporo 001-0020, Japan \\ (Received 19 November 2008; published 6 April 2009)
}

\begin{abstract}
Heterogeneity is one of the most important and ubiquitous types of external perturbations in dissipative systems. To know the behaviors of pulse waves in such media is closely related to studying the collision process between the pulse and the heterogeneity-induced-ordered pattern. In particular, we focus on unidirectional propagation of pulses in a medium with an asymmetric bump heterogeneity. This topic has attracted much interest recently with respect to potential computational aspects of chemical pulse propagation as well as with respect to pulse propagation in biological signal processing. We employ a three-component reactiondiffusion system with one activator and two inhibitor species to illustrate these issues. The reduced dynamics near a drift bifurcation describes the phenomena in the full partial differential equations by ordinary differential equations. Such a reduced dynamics is able to capture unidirectional propagation properties of pulses near an asymmetric heterogeneity in a qualitatively correct way. A remarkable feature is that such unidirectional behavior is linked to the imperfection of global bifurcation structure and the resulting asymmetric locations of critical points.
\end{abstract}

DOI: 10.1103/PhysRevE.79.046205

PACS number(s): 82.40.Bj, 68.35.Fx, 82.20.Wt, 89.75.Kd

\section{INTRODUCTION}

Spatially localized moving patterns such as traveling pulses and fronts in one dimensions and traveling spots in higher dimensions are fundamental objects arising in many dissipative systems and display a large variety of dynamical behaviors $[1-5]$. The propagation manner of such waves in heterogeneous media is of great relevance to the understanding of information exchange in chemical and biological systems. Stationary and oscillatory pinning as well as reflection and diffraction by heterogeneities have been reported [6-19]. Related phenomena occur upon sudden change in the geometry in two or three dimensions: fronts and pulses may be reflected near exits of narrow channels $[20,21]$ because sudden extensions lead to wave curvature and effective changes in the diffusion constant [22]. A variety of outputs is observed when wave encounters a heterogeneity typically depending on quantities such as the height and width of the obstacle. Possible behaviors of pulses and other waves are rebound, stationary, and oscillatory pinning at the heterogeneity as well as penetration. A remarkable observation is that the full partial differential equation (PDE) dynamics can be reduced to finite-dimensional ordinary differential equation (ODE) dynamics near a drift bifurcation. An analogous approach with an ODE based reduced dynamics has been given for the behavior of periodic traveling patterns in media with broken symmetry $[23,24]$. Recently, considerable effort has been devoted to analyze the computational potential of pulse waves in chemical logic and switching devices [25-28]. In such reaction-diffusion processors, data are represented by concentration profiles of chemical species and computation is performed by propagation and interaction of pulse waves. One particularly exciting perspective comes from the dramatic increase in research on unconventional computing architectures by using traveling chemical waves in a sensible and programmable way. A few studies have provided interesting examples of chemical diodes obtained by controlling the geometrical arrangement of media, in which the rectification of chemical waves was observed in light-sensitive Belousov-Zhabotinsky medium with asymmetric light illumination [29-31]. The spatial heterogeneity in the light intensity distribution is crucial to determine the direction of unidirectional propagation even in one-dimensional system [32]. Earlier work on simulations of chemical pulses on spatially heterogeneous catalytic surfaces has also revealed unidirection propagation $[33,34]$. Although numerous experiments have been carried out, the mechanism of how the asymmetry of heterogeneity causes the unidirectional motion is not well understood. Resolution of these issues may also add to the understanding of the processing of biological signals such as action potentials in the brain, which are typical examples of reaction-diffusion pulses. In many biological systems, it is crucial how reaction-diffusion waves react to external perturbations such as heterogeneous concentrations of activating factors [35-39].

In this paper, we focus on dynamics of traveling pulses that encounter heterogeneities of asymmetric bump type for a three-component reaction-diffusion system with one activator and two inhibitors. Especially we study how the asymmetry of heterogeneity causes unidirectional pulse propaga- 

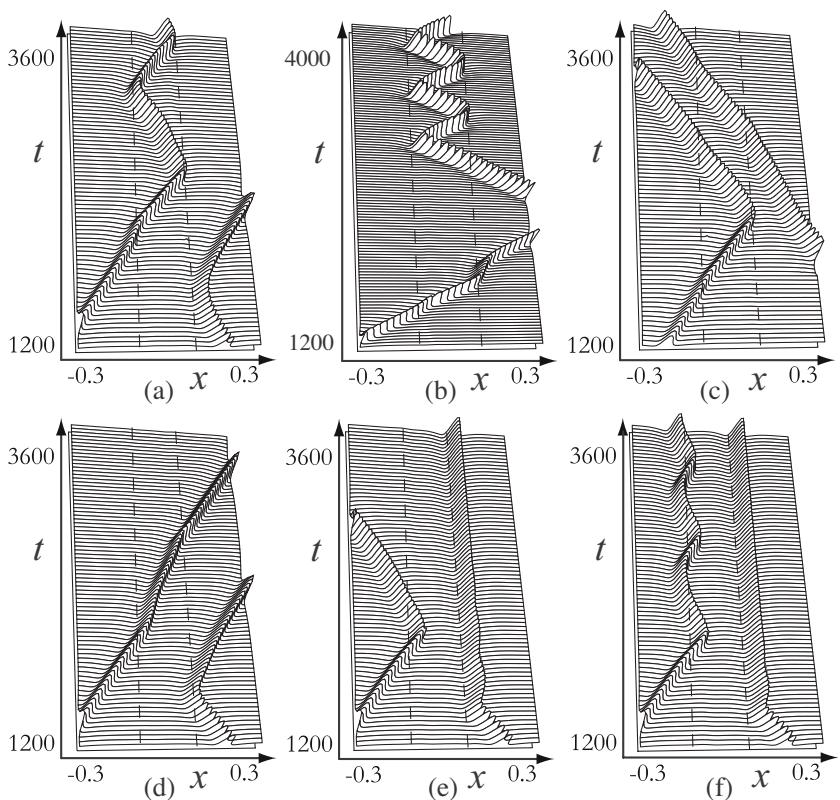

FIG. 1. The typical spatiotemporal patterns of unidirectional pulse behaviors for PDE system are depicted in (a) unidirectional pinning of pulses coming from the left-side end for $\epsilon=0.10$, (b) unidirectional oscillatory pinning from the right for $\epsilon \approx 0.064$, (c) rectification to the left-side end for $\epsilon=0.05$, (d) rectification to the right for $\epsilon=-0.05$, (e) unidirectional pinning from the right for $\epsilon$ $=-0.10$, and (f) unidirectional oscillatory pinning for $\epsilon=-0.20$. In this paper, we focus on the collision process between traveling pulses and defects induced by a bump heterogeneity. Interactions between pulses are neglected in the analysis described below. We employ the following parameter values $d=0.15, k_{1}^{R}=-6.50, k_{2}$ $=2.0, \quad k_{3}=1.0, \quad k_{4}=8.5,\left(D_{u}, D_{v}, D_{w}\right)=\left(0.9 \times 10^{-4}, 1.0 \times 10^{-3}, 1.0\right.$ $\times 10^{-2}$ ), and $\tau=40.0$.

tion phenomena as the ones surveyed in Fig. 1. Here such an asymmetry is imposed by different slopes at both ends of the bump. Since we introduce the heterogeneity in an additive way, the uniform background state is not a solution anymore and is replaced by a nonuniform stationary pattern, which we henceforth call defect. Such a defect is not uniquely determined for a given heterogeneity. In fact, there are many candidates even for the simple heterogeneity of jump type [40]. In this paper, we employ the defect with smallest amplitude as the new background state. When the pulse encounters the bump heterogeneity, a variety of outputs, including pinning, rebound, and penetration, is observed. Transitions among those outputs occur as the width and the height of the bump are varied. These behaviors can be regarded as a result of collision between a pulse and a defect. The method developed in Refs. [41-44] for symmetric obstacles is employed here in order to clarify the underlying mechanisms also for asymmetric heterogeneities. The basic method employed in those references is to find the origin of the sorting mechanism, by which the output can be controlled based on the information of the local dynamics near the so-called scattors, i.e., a special class of unstable solutions which link input to output at the collision point [40]. A reduction from PDE to finite-dimensional ODEs is also possible near the singularity, and the resulting system inherits most of the essential dy- namics from the original PDE. It turns out that there are various types of heterogeneity-induced localized patterns, i.e., defects around the bump heterogeneity, which are captured by the critical points of reduced ODEs. The basin of each critical point and the switching among those basins depending on parameters explain all the transitions of the original PDEs; in fact, various bifurcations such as Hopf and homoclinic ones cause the onset of those transitions. The reduction method permits us to clarify the mathematical structure controlling the dynamic transitions in a precise way, especially the asymmetry of bump causes, for instance, the imperfection of the bifurcation structure and the transition from a heteroclinic to a homoclinic one, resulting in unidirectional motions.

The paper is organized as follows. In Sec. II, we introduce our model system and the precise form of bump heterogeneity. A reduction to ODEs and comparison of phase diagrams between PDE and ODE dynamics are presented. In Sec. III, we show that the reduced ODEs clarify how dynamic transitions occur depending on the width and height of the bump. The colliding dynamics between pulses and defects for symmetric case was extensively studied for both moderate and steep bumps $[45,46]$, in which we discussed three types of underlying mechanisms causing a variety of input-output relation and their transitions in heterogeneous media. The first one is simply due to the local bifurcation of attractors, for instance, a fixed point of ODEs is switched to a limit cycle, i.e., the Hopf bifurcation changes a stationary pulse into an oscillatory pinning behavior. The second is that the global bifurcation of the limit cycle is responsible for the onset of the pinning-depinning transition of pulse behaviors. In fact, such a global bifurcation typically accompanies a saddlenode bifurcation and its unstable part of the branch blocks the entering of orbits into the pinning state when the parameter passes the global bifurcation point.

Third, it turns out that hidden unstable patterns located at infinity and their stable and unstable manifolds play crucial roles to direct the orbital behaviors in heterogeneous media. Section III highlights how those mechanisms are changed or modified for an asymmetric heterogeneity. One main goal is to understand the onset of unidirectional behaviors that do never occur in the symmetric case. The outcome can be summarized by emphasizing three crucial points: imperfection of the bifurcation structure, the change in a global bifurcation from a heteroclinic to a homoclinic one, and order in which Hopf and global bifurcation points appear. All behaviors encountered in simulation of the PDE are qualitatively reproduced by the reduced ODE dynamics and are therein organized by the aforementioned three guiding facts.

In this paper we concentrate on the study of the reduced ODE dynamics for the narrow bump case with three critical points. As the width is increased, the number of critical points is increased from three to five, and there occurs a subtle bifurcation of the limit cycles emanated from multiple critical points. In fact, they form trouserslike structure with respect to the bump height, in which a figure-of-eight orbit appears as in Refs. [45,46]. In the Appendix, we will show the attractor switching behavior obtained for the wide bump case and discuss about imperfection of the figure-of-eight (double homoclinic, ambiclinic) bifurcation caused by an asymmetry. 


\section{MODEL AND THEORY}

\section{A. Three-component reaction-diffusion system}

In order to investigate the dynamics of traveling pulses in heterogeneous media, we employ the following threecomponent reaction-diffusion system, which was studied as a qualitative model of gas discharge phenomena $[2,4,47]$,

$$
\begin{gathered}
u_{t}=D_{u} \triangle u+k_{2} u-u^{3}-k_{3} v-k_{4} w+k_{1}, \\
\tau v_{t}=D_{v} \triangle v+u-v, \\
w_{t}=D_{w} \triangle w+u-w,
\end{gathered}
$$

where $\triangle$ is the Laplacian, $u=u(t, x), v=v(t, x)$, and $w$ $=w(t, x)$ depend on time $t$ and $x \in \mathbb{R}^{1}, k_{1}, k_{2}, k_{3}$ and $k_{4}$ are kinetic parameters, and $\tau$ and the diffusion coefficients $D_{u}, D_{v}, D_{w}$ are positive constants.

System (1) can be regarded as a generalized FitzHughNagumo type of model, namely, it is a representative model which supports various spatially localized waves, especially traveling pulses and spots in one-, two-, or three-spatial dimensions; in fact, the second inhibitor $w$ is indispensable for the coexistence of multiple number of stable traveling spots in two- and three-dimensional spaces. The role of the first inhibitor $v$ in two or three dimensions is to stabilize the back end of a traveling spot, while the second inhibitor $w$ prevents a lateral growth of a traveling spot into a double pair of spirals. The latter behavior is typical for two-component activator-inhibitor systems. System (1) is a simple and a prototypical model for the study of interaction among moving particle patterns in dissipative systems. It was studied in [40-42] under the parameter setting used here that Eq. (1) becomes an excitable system with unique stable equilibrium point and has a stable traveling pulse. The velocity of the pulse is an increasing function of $k_{1}$.

In this study, we consider the pulse dynamics in asymmetric bump-shaped inhomogeneous media as shown in Fig. 2, especially how such dynamics depend on the height $\epsilon$ and the width $d$ of bump. We introduce the following asymmetric heterogeneity function $\chi(x, d)$ to parameter $k_{1}$ as $k_{1}(x)=k_{1}^{R}$ $+\epsilon \chi(x, d)$, where

$$
\chi(x, d)=\frac{1}{1+e^{-\gamma^{L}(x+d / 2)}}+\frac{1}{1+e^{+\gamma^{R}(x-d / 2)}}-1,
$$

and $\epsilon \equiv k_{1}^{M}-k_{1}^{R}$. Numerically, we can set $k_{1}(-l / 2)=k_{1}(l / 2)$ $=k_{1}^{R}$ and $k_{1}(0)=k_{1}^{M}$ for an appropriate system size. The parameters $\gamma^{L, R}$ control the steepness of the slopes around the left and right jump points of $x= \pm d / 2$, respectively. Note that the above heterogeneity satisfies the relation $\chi(-x,-d)$ $=-\chi(x, d)$. An advantage of functional form (2) is that the left-right reversal form of the heterogeneity can be obtained by changing the sign of $d$ and $\epsilon$ at the same time. Hereafter, we set to $\left(\gamma^{L}, \gamma^{R}\right)=(100.0,1000.0)$, i.e., right is steeper than left.

After we introduce spatial heterogeneity to the kinetic coefficient of an additive type, the uniform state is no more a background state for the resulting system, several inhomogeneous steady states are observed for each $(d, \epsilon)$. In this paper, we employ the smallest defect in amplitude as the background state for the heterogeneous system. When a stable traveling pulse approaches the bump-shaped regime, it collides with small defects, so our problem can be regarded as the collision problem between the pulse and defect.

The symmetric bump case $\left(\gamma^{L}=\gamma^{R}\right)$ was studied in the previous paper $[40,45,46]$ in which left- and right-going traveling pulses have the same behaviors. This is not the case for the asymmetric case. It contains a variety of unidirectional behaviors, including rectification and unidirectional pinning behaviors, as in Fig. 1 in which the bump height $\epsilon$ is decreased. Figure 1(c) shows that rebound of a pulse at the steep side of the bump domain is found for a small absolute $\epsilon$ and the counterpart at the moderate side is penetration. The resulting one-way traffic of pulse propagation is sometimes called a rectification phenomenon. When the sign of $\epsilon$ is changed, i.e., bump or dent, the left-right reversal behavior is obtained as shown in Fig. 1(d). Figures 1(a), 1(b), and 1(e) show the unidirectional pinning behaviors, in which a pulse coming from one side is trapped around bump domain, while that from the another side is rebound or penetration. The pinning direction depends on the $\epsilon$ value. As depicted in Fig. 1 (f), the unidirectional oscillatory pinning behavior appears by still decreasing $\epsilon$, in which pulses are trapped at both sides of bump domain, but one of them shows the oscillatory motion. The details of unidirectional behaviors will be discussed in Sec. III. The above findings suggest that an asymmetric heterogeneity with variable height (or width) can play the role of a dynamical transition that depending on its state selects the direction of possible pulse propagation. As mentioned in our previous paper, the ODE reduction method is not limited to the symmetric case; the analysis for the asymmetric case can be done in a parallel way. We now want to see what happens when such an asymmetry is present, focusing on understanding not only the process that leads to pinning-depinning transition but also the bifurcation pictures that lead to unidirectional behaviors of pulses.

\section{B. Reduction to ODEs}

The pulse dynamics in heterogeneous media can be reduced to a finite-dimensional one when the associated parameter values are close to the drift bifurcation of $k_{1}=k_{1}^{c}$, namely, the pulse velocity is slow [45,48-51]. Considering the original PDE of Eq. (1) in a neighborhood of $k_{1}=k_{1}^{c}$, with new parameter $\eta$ such that $k_{1}=k_{1}^{c}+\eta$,

$$
\begin{aligned}
\boldsymbol{u}_{t}= & D \boldsymbol{u}_{x x}+F\left[\boldsymbol{u} ; k_{1}(x)\right]=\mathcal{L}\left(\boldsymbol{u} ; k_{1}^{c}\right)+[\eta+\boldsymbol{\epsilon} \chi(x, d)] \boldsymbol{g}(\boldsymbol{u}), \\
& t>0, x \in \mathbb{R},
\end{aligned}
$$

where $\boldsymbol{g}$ is $N$-dimensional vector-valued functions. For our system $(1), \boldsymbol{g}=(1,0,0)^{t}$. Let $X:=\left\{L^{2}(\mathbb{R})\right\}^{N}, \boldsymbol{u}=\left(u_{1}, \ldots, u_{N}\right)^{T}$ $\in X$ be an $N$-dimensional vector, $D=\operatorname{diag}\left(D_{1}, \ldots, D_{N}\right)$, $F: \mathbb{R}^{N} \rightarrow \mathbb{R}^{N}$, and $\chi(x, d)$ be a $C^{1}$ function. We assume that there exists a $k_{1}=k_{1}^{c}$ such that the nontrivial standing pulse solution $S\left(x ; k_{1}\right)$ of Eq. (1) exists, i.e., $\mathcal{L}\left(S ; k_{1}^{c}\right)=0$.

Let $L=\mathcal{L}^{\prime}\left(S ; k_{1}^{c}\right)$ be the linearized operator of Eq. (3) with respect to $\boldsymbol{u}$ at $\boldsymbol{u}=S\left(x, k_{1}^{c}\right)$. $L$ has a singularity at $k_{1}=k_{1}^{c}$ consisting of drift bifurcation in addition to the translation-free 


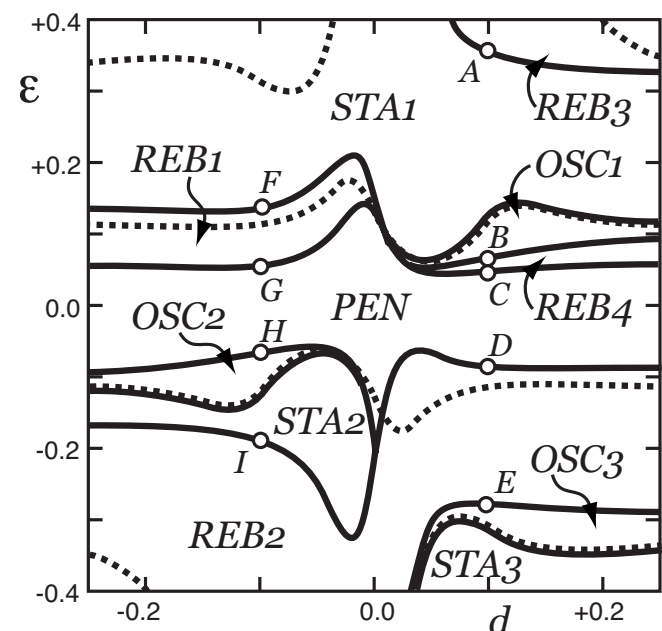

(a) ODE

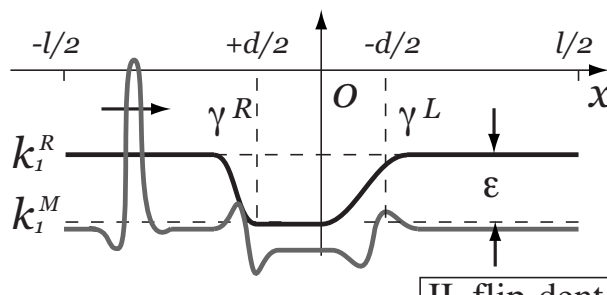

II. flip-dent

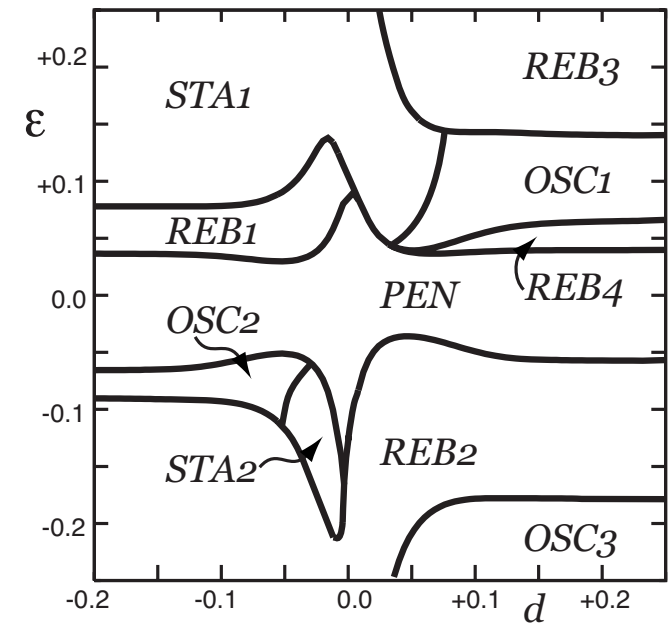

(b) PDE

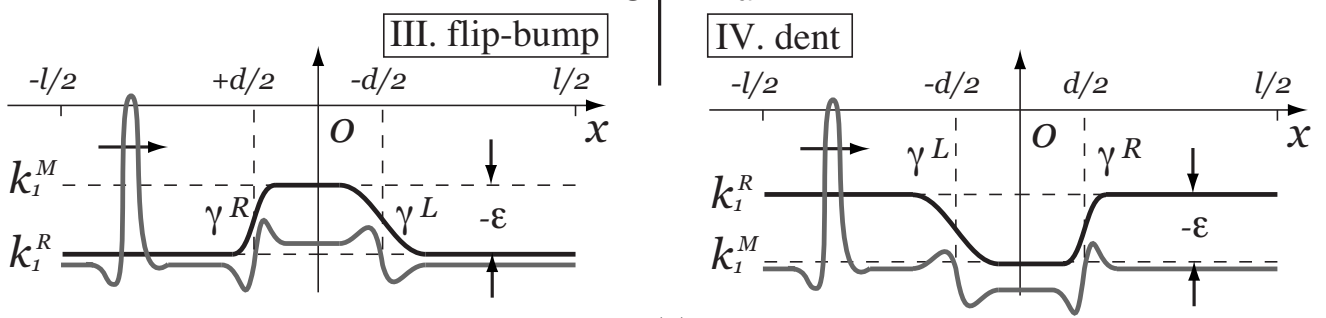

(c)

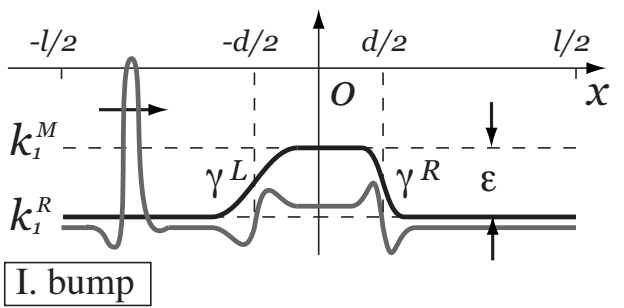

I. bump

FIG. 2. (a) Phase diagram of ODE dynamics for the asymmetric case of $\left(\gamma^{L}, \gamma^{R}\right)=(50,100)$. There are 11 type behaviors: those phase boundaries are indicated by solid lines. The dotted lines correspond to the Hopf bifurcation lines for the equilibrium points. (b) Phase diagram of PDE dynamics for the asymmetric case of $\left(\gamma^{L}, \gamma^{R}\right)=(100,1000)$. (c) Schematic picture of initial condition for asymmetric heterogeneous bump case. Solid line shows how $k_{1}(x)$ changes in spatial direction, and gray line displays a manner how a traveling pulse hits a small defect created by the heterogeneity for the first (fourth) quadrant of $d>0$ and $\epsilon>(<) 0$ named as case I (bump) [case IV (dent)]. The $k_{1}(x)$ manner in the negative $d$ region looks just like a left-right flip type of $d>0$.

zero eigenvalue. That is, there exist two eigenfunctions $\phi(x)$ and $\psi(x)$ such that $L \phi=0$ and $L \psi=-\phi$, where $\phi=\partial S / \partial x$. Note that $\phi(x)$ and $\psi(x)$ are odd functions. $\psi(x)$ represents the deformation vector with Jordan form for the drift bifurcation. Similar properties also hold for $L^{*}$. That is, there exist $\phi^{*}$ and $\psi^{*}$ such that $L^{*} \phi^{*}=0$ and $L^{*} \psi^{*}=-\phi^{*}$, where $\phi^{*}(x)$ and $\psi^{*}(x)$ are odd functions. Let $E=\operatorname{span}\{\phi, \psi\}$ and the eigenfunctions are normalized by $\langle\psi, \phi\rangle=\left\langle\psi, \psi^{*}\right\rangle=0$ and $\left\langle\phi, \psi^{*}\right\rangle=1$.

The movement of a pulse solution $\boldsymbol{u}$ is essentially described by the scalar functions $p(t), q(t) ; p$ denotes the location of pulse and $q$ for its velocity. For small $\epsilon$ and $\eta$, we can approximate a solution $\boldsymbol{u}$ by

$$
\boldsymbol{U}=S(x-p)+q \psi(x-p)+q^{2} \zeta_{1}(x-p)+q \eta \zeta_{2}(x-p),
$$

where the remaining terms $\zeta_{1}, \zeta_{2} \in E^{\perp}$ are defined by $L \zeta_{1}$ $+F^{\prime \prime}(S) \psi^{2} / 2+\psi_{x}=0$ and $L \zeta_{2}+g(S)=0$. Substituting Eq. (4) into Eq. (3) and taking inner product with $\phi^{*}$ and $\psi^{*}$, we obtain the principal part by the following system:

$$
\begin{gathered}
\dot{p}=q-\epsilon \Gamma_{0}(p, d), \\
\dot{q}=M_{1} q^{3}+M_{2} q \eta+\epsilon \Gamma_{1}(p, d),
\end{gathered}
$$

where

$$
M_{1}=\left\langle F^{\prime \prime}(S) \psi \cdot \zeta_{1}, \phi^{*}\right\rangle+\frac{1}{6}\left\langle F^{\prime \prime \prime}(S) \psi^{3}, \phi^{*}\right\rangle+\left\langle\partial_{x} \zeta_{1}, \phi^{*}\right\rangle,
$$




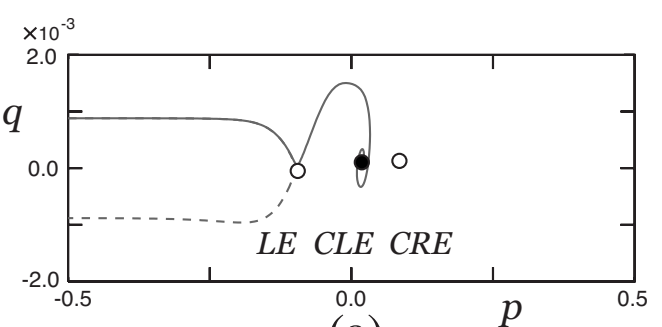

(a)

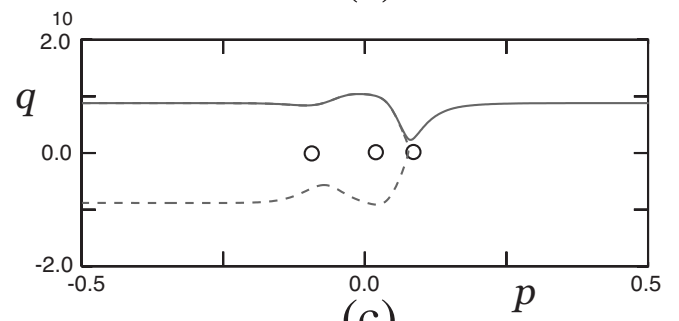

(c)

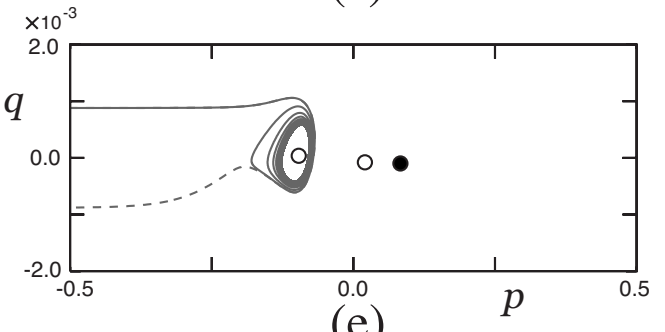

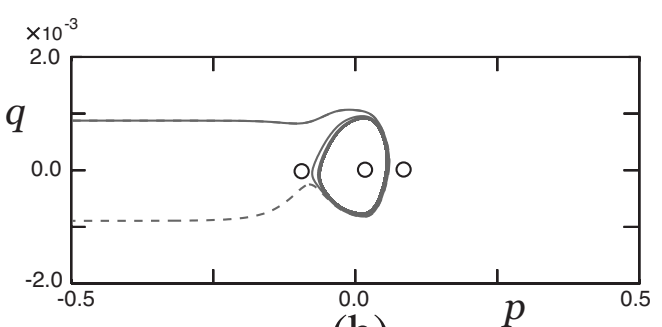

(b)

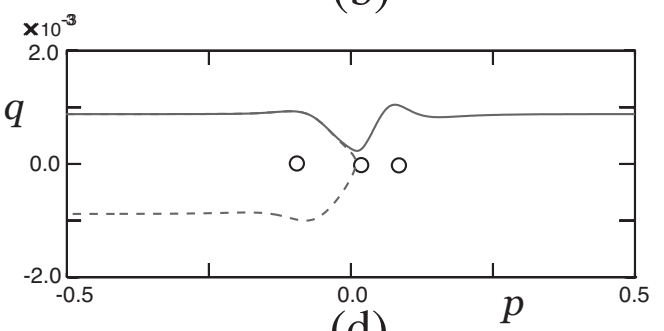

(d)

FIG. 3. Orbit flows in $(p, q)$ space for $d=+0.10$ are depicted in (a) for phase boundary between REB3 and STA1 around $\epsilon \approx 0.357$ near point $A$ in Fig. 2(b), (b) for transition from OSC1 to REB4 around $\epsilon \approx 0.061$ near point $B$, (c) for transition from REB4 to PEN around $\epsilon$ $\approx 0.050$ near point $C,(\mathrm{~d})$ for transition from PEN to REB2 around $\epsilon \approx-0.067$ near point $D$, and (e) for transition from REB2 to OSC3 around $\epsilon \approx-0.274$ near point $E$. The solid and open circles indicate the stable and unstable stationary states, respectively.

$$
M_{2}=\left\langle F^{\prime \prime}(S) \psi \cdot \zeta_{2}, \phi^{*}\right\rangle+\left\langle\partial_{x} \zeta_{2}, \phi^{*}\right\rangle+\left\langle g^{\prime}(S) \psi, \phi^{*}\right\rangle,
$$

and

$$
\begin{aligned}
& \Gamma_{0}(p, d)=\int_{-\infty}^{\infty} \chi(x, d) g[S(x-p)] \cdot \psi^{*}(x-p) d x, \\
& \Gamma_{1}(p, d)=\int_{-\infty}^{\infty} \chi(x, d) g[S(x-p)] \cdot \phi^{*}(x-p) d x .
\end{aligned}
$$

Here the coefficients $M_{1}, M_{2}$ and heterogeneous term $\Gamma_{i}(p, d)$ depend on the model system and influence a lot over the dynamics. The effect of heterogeneity becomes acceleration (deceleration) when $\epsilon \Gamma_{1}>(<) 0$. For our system (1) with $k_{1}^{c} \approx-6.79$, they are computed as $M_{1} \approx-11451<0$ and $M_{2} \approx 0.031>0$ from the profiles of eigenfunctions and profiles of $\Gamma_{i}$ are obtained numerically as in Figs. 5(a)-5(c). A more complete treatment was shown in [45].

For the homogeneous case of $\epsilon=0$, the dynamics is reduced to study a single equation of second order independent of $p$. The equilibrium point $E_{1}$ of $q=0$ corresponds to the standing pulse solutions and $E_{2}^{ \pm}$given by $q= \pm \sqrt{-M_{2} \eta / M_{1}}$ for $\eta>0$ correspond to the right- and left-going traveling pulses. As $\eta$ is changed from negative to positive, the drift bifurcation occurs and $E_{2}^{ \pm}$of $q \neq 0$ bifurcate from $E_{1}$ supercritically.
Since we are interested in the regime in which stable traveling pulses exist, we employ $\eta=+0.29$ for definiteness, i.e., $k_{1}^{R}=k_{1}^{c}+\eta$ is set to -6.50 . We will use this value throughout this paper, which implies that $E_{2}^{ \pm}$of $q$ is $\approx \pm 8.8 \times 10^{-4}$. It should be noted that the results in the sequel are also valid for smaller $\eta>0$. Since $\Gamma_{i}$ converges to 0 as $p \rightarrow \pm \infty$, the orbit converges to either $E_{2}^{+}$or $E_{2}^{-}$as $t \rightarrow \infty$ in a far field depending on the sign of $q(0)$. In fact, the far-field limit of Eq. (5) is given by its second equation without the $\Gamma_{1}$ term equivalent to the homogeneous case. The issue is to study the fate of the orbit starting from $E_{2}^{+}$at $p=-\infty$ for a given bump heterogeneity. When it goes to $E_{2}^{+}\left(E_{2}^{-}\right)$as $p \rightarrow+\infty$, it means that the pulse penetrates (rebounds from) the bump. Depending on the width and the height of the bump, the orbit could be trapped by one of the pinning states around the bump as will be discussed in Sec. II C. In general what we have to do is to find a heteroclinic connection (in a generalized sense) starting from $E_{2}^{+}$at $p=-\infty$.

\section{Typical flows of ODEs and the symmetry of heterogeneous function}

The issue here is to study the fate of the orbit starting from $E_{2}^{+}$at $p=-\infty$ for a given bump heterogeneity. As it is expected, penetration occurs when the difference $|\epsilon|$ is small, i.e., pulses can go across the bump. We find up to 11 distinct regions in the phase diagram with four different types of fundamental behaviors, depending on the width $d$ and the 


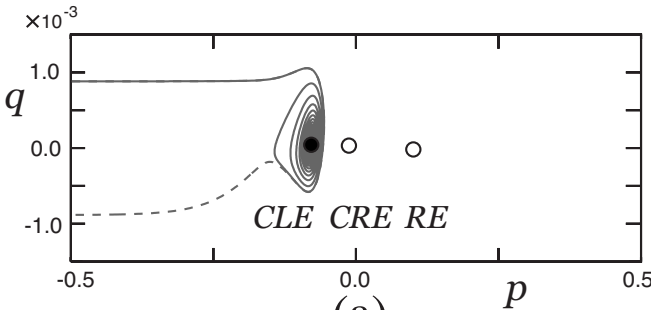

(a)

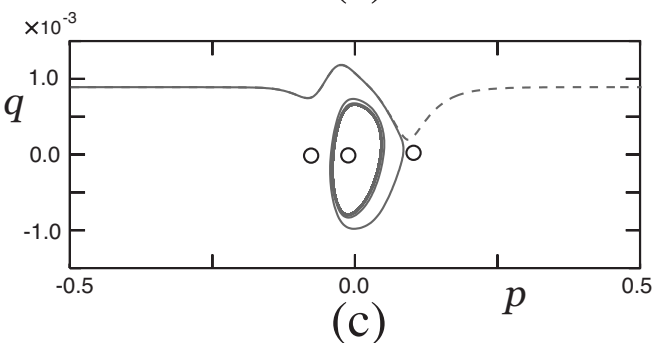

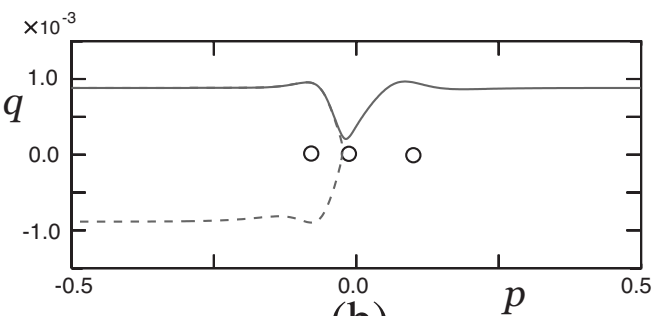

(b)

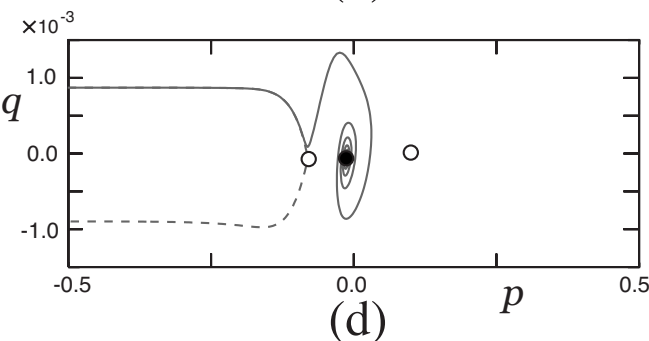

FIG. 4. Orbit flows in $(p, q)$ space for $d=-0.10$ are depicted in (a) for transition from STA1 to REB 1 around $\epsilon \approx 0.124$ near point $F$ in Fig. 2(b), (b) for transition from REB1 to PEN around $\epsilon \approx 0.047$ near point $G$, (c) for transition from PEN to OSC2 around $\epsilon \approx-0.073$ near point $H$, and (d) for transition from STA2 to REB2 around $\epsilon \approx-0.182$ near point $I$. The solid and open circles indicate the stable and unstable stationary states, respectively.

height $\epsilon$ of the heterogeneity. The fundamental types of behavior for a single pulse include penetration of the heterogeneity (PEN), stationary (STA), and oscillatory pinning (OSC) of the pulse at the heterogeneity and reflection (rebound) of the pulse at the obstacle (REB). Detailed results for the reduced ODE dynamics are shown in Fig. 2(a), which reflects the original PDE dynamics quite well [see Fig. 2(b)]. The comparison between the ODE and PDE phase diagrams for the collision dynamics between pulse and defect in Fig. 2 shows good qualitative agreement. Note that the asymmetry of the heterogeneities breaks the $d \rightarrow-d$ symmetry found earlier for symmetric heterogeneities (see Remark II.1). Figures 3 and 4 show all the cases of orbit flows on the phase boundaries marked by the characters $(A-I)$ in Fig. 2(a).

Most parts of the diagram still have the look of those for the symmetric cases of Fig. 7 in [45]. What is expected after the penetration for $d>0$, as $\epsilon$ is decreased for the situation of case I-bump in Fig. 2(c)—is that some sort of rebound by the second collision with the steep side. On the other hand, for $\epsilon<0$ for case IV-dent situation-the onset of the pinning-depinning transitions dominates the dynamics, hence the trapping inside the bump occurs. However, the steepness of the right side greatly also influences the pulse behaviors and there are still several qualitative differences from the symmetric case. One is that rebound behavior (REB4) is observed between PEN and OSC1. The REB4 region is embedded in $\mathrm{PEN}$ region because right-going pulse passed the moderate left side will face the right side: if the right side is the same moderate slope as the left one, pulse will penetrate the bump just as the symmetric case; if the right side slope is steeper, pulse will rebound. Figures 4(b) and 4(c) show us the orbit flow for the transitions from OSC1 to REB4 and from REB4 to PEN. An asymmetric heterogeneity breaks the reflection symmetry around the origin and, hence, the depinning mechanism subject to asymmetry causes qualitative differences to the symmetric cases. In particular, this REB4 phase is crucial to the rectification behavior of pulses of Fig. 1(b), as will be discussed in Sec. III A. We note that, when $d=0$, i.e., $\chi(x, 0)$ is not a zero, there are small defects around the origin and the translational invariance is broken, so we cannot always observe penetration behavior unlike the symmetric case. As $|\epsilon|$ is increased, the behavior depends on the sign of $\epsilon$. More detailed analysis of ODEs allows us to explain all the dynamic transitions in a precise way. In fact, it turns out that scattors and their stable and unstable manifolds play a crucial role for our purpose as shown in Figs. 3 and 4.

Remark II.1. Here, unlike the symmetric case, we also explore the parameter space for the negative $d$ region. As shown in Fig. 2(c), in the positive $d$ region of Fig. 2(a), the right-going pulse coming from $p=-\infty$ collides firstly moderate side of bump. The first quadrant of $\epsilon>0$ is the bump case and the forth quadrant is the corresponding dent case of $\epsilon$ $<0$. Remind that the asymmetric heterogeneity used here holds the relation of $\chi(-x,-d)=-\chi(x, d)$. In the negative $d$ area of the phase diagrams of Fig. 2, the orbit collides first steep side of bump. The third quadrant of $\epsilon<0$ to the flipbump case as shown in Fig. 2(c) and the second quadrant of $\epsilon>0$ correspond to the corresponding flip-dent case. Substituting it into the integral forms of Eq. (6), we can easily obtain the relation of $\Gamma_{i}(-p,-d)=\Gamma_{i}(p, d)$, as required by the symmetry of our system with respect to reflection in $(p, d)$. Here we use that $g$ is constant and $\psi^{*}$ and $\phi^{*}$ are odd functions. This leads us to find that the ODE dynamics with the sign change in $(-d,-\epsilon)$,

$$
\begin{gathered}
\dot{p}=q+\epsilon \Gamma_{0}(p,-d), \\
\dot{q}=M_{1} q^{3}+M_{2} q \eta-\epsilon \Gamma_{1}(p,-d),
\end{gathered}
$$

is equivalent to Eq. (5) under the transformations $(p, q) \rightarrow$ $(-p,-q)$. This means that the exact same things happen in both trajectories starting from $E_{2}^{-}$at $p=+\infty$ and from $E_{2}^{+}$at $p=-\infty$ if we make the change in variables $(d, \epsilon) \rightarrow(-d,-\epsilon)$. 

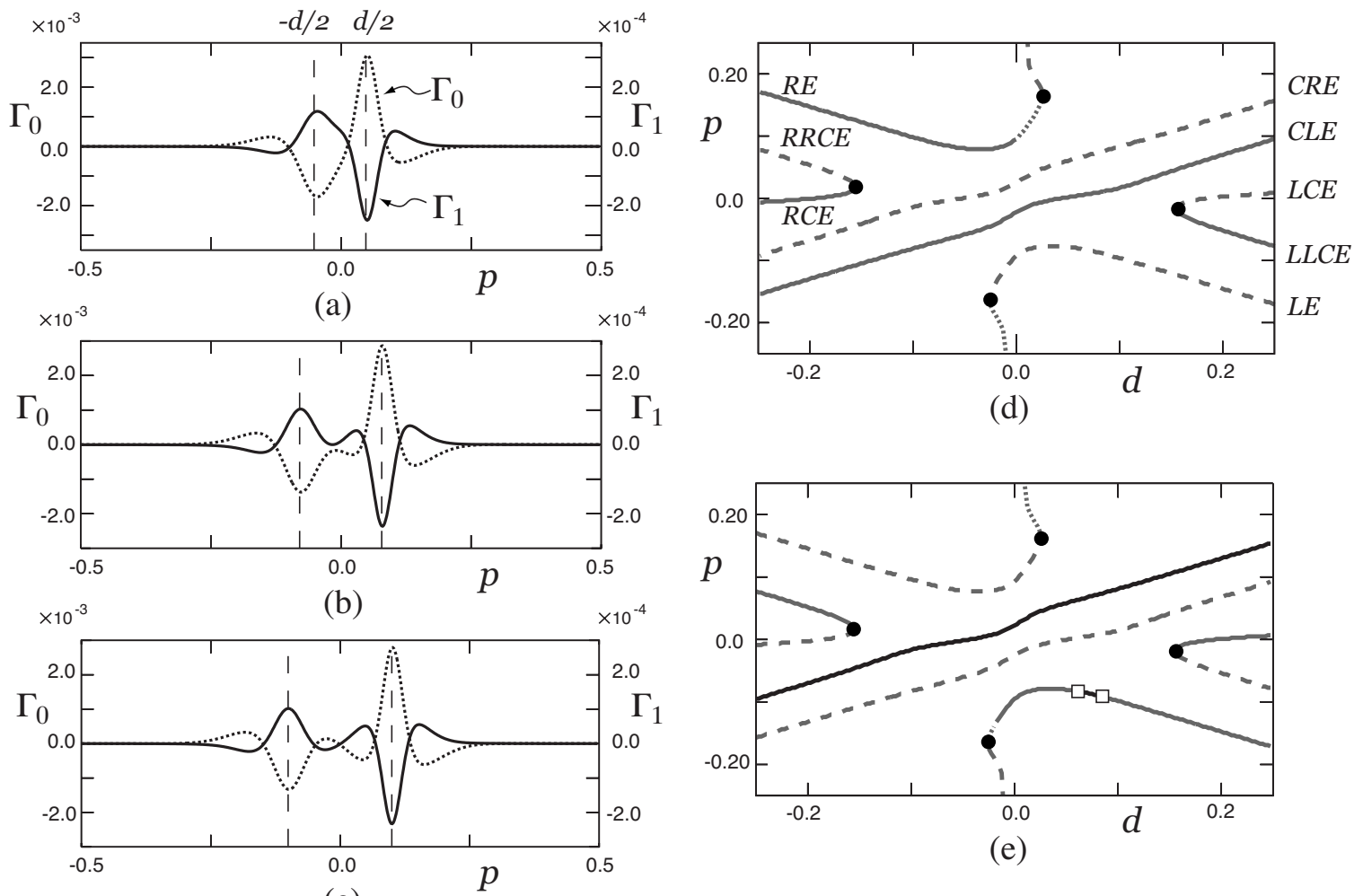

(d)

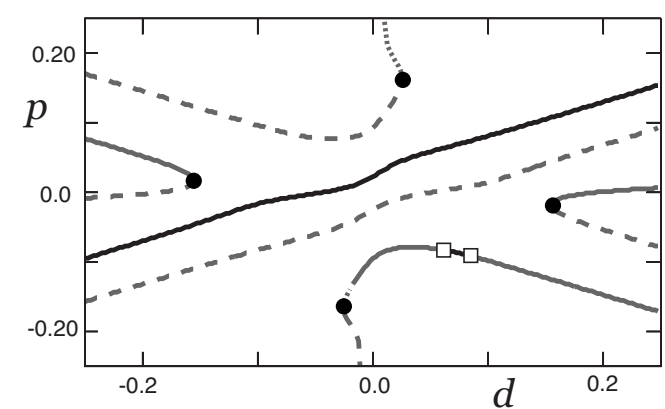

(e)

(c)

FIG. 5. The profiles of the heterogeneity functions $\Gamma_{i}$ for the asymmetric bump are shown in (a) for $d=0.10$, (b) for $d \approx 0.16$, and (c) for $d=0.20$. The dotted and solid lines correspond to $\Gamma_{0}$ and $\Gamma_{1}$, respectively. The asymmetry of the system induces an imperfection of pitchfork bifurcations. By increasing the bump width $d$, the number of zero points increases pairwise through the saddle-node bifurcations. The black circles indicate the saddle-node bifurcation points. When $\epsilon$ changes its sign, the properties of the fixed points are exchanged between focus and saddle. Bifurcation diagrams of ODE system with respect to the bump width $d$ for $\epsilon=0.055$ and -0.30 are shown in (d) and (e), respectively. Note that the narrow node regimes appear just before the focus regimes near the limiting points. The black and gray solid lines indicate the stable and unstable foci and the gray broken and dotted lines indicate the saddle and unstable nodes, respectively.

\section{RESULTS AND DISCUSSIONS}

\section{A. Heterogeneous function and bifurcation diagram for width $d$}

We first study the existence and stability of equilibria for ODE system (5). The location of equilibrium point is reduced to find the solution of $M_{1} \epsilon^{2} \Gamma_{0}(p, d)^{3}+M_{2} \eta \Gamma_{0}(p, d)$ $+\Gamma_{1}(p, d)=0$. For sufficiently small $\epsilon$ and $\eta$, the last term becomes the leading order; therefore it suffices to focus on the behaviors of $\Gamma_{1}$. It is numerically confirmed that $\Gamma_{1}$ has three zeros for small $|d|$ and five zeros for large $|d|$ as in Figs. 5(a) and 5(c). The critical case occurs when the horizontal line becomes tangent to the local minimum of $\Gamma_{1}$ as shown in Fig. 5(b), and the number of zero points of $\Gamma_{1}$ is increased from three to five via saddle-node bifurcation as $d$ is increased. For three zeros case, the middle one is located inside the bump and the other two are outside of the bump. Note that the $\Gamma_{i}$ functions are no longer odd functions due to the asymmetry of the bump. $\Gamma_{i}$ has the largest extremum at the steeper side of bump and smaller variation at moderate side, which comes from the property that $\Gamma_{i} \searrow 0$ as $\gamma \searrow 0$. Recalling that $q$ is the velocity of the pulse, the positive (negative) part of $\Gamma_{1}$ represents acceleration (deceleration) for positive $\epsilon$. Therefore, when the pulse comes from the left, the pulse feels like small deceleration $\rightarrow$ large acceleration $\rightarrow$ huge deceleration $\rightarrow$ small acceleration and vice versa with opposite effect for negative $\epsilon$. Therefore the effect of the huge deceleration region around steep side becomes visible primarily as $\epsilon$ is increased from zero, and hence rebound (REB4) occurs instead of penetration. As $\epsilon$ is still increased, transition from REB4 to OSC1 occurs since pulses are trapped between large acceleration and huge deceleration regions. Even larger $\epsilon$, the first small deceleration regime becomes strong enough to push back the pulse and the transition from OSC1 to REB3 occurs.

The stability property of equilibrium point of $(p, q)$ $=(\bar{p}, \bar{q})$ is determined by the following linearized system:

$$
\left(\begin{array}{c}
\dot{p} \\
\dot{q}
\end{array}\right)=\left.\left(\begin{array}{cc}
-\epsilon \partial_{p} \Gamma_{0} & 1 \\
\epsilon \partial_{p} \Gamma_{1} & 3 M_{1} \epsilon^{2} \Gamma_{0}^{2}+M_{2} \eta
\end{array}\right)\right|_{(\bar{p}, \bar{q})}\left(\begin{array}{l}
p \\
q
\end{array}\right) \equiv \widetilde{L}\left(\begin{array}{l}
p \\
q
\end{array}\right) .
$$

The eigenvalue problem $\tilde{L} \Psi=\lambda \Psi$ can be solved easily as $\lambda=\frac{1}{2}\left(\operatorname{tr}(\widetilde{L}) \pm \sqrt{\left.\operatorname{tr}(\widetilde{L})^{2}-4 \Delta\right)}\right.$, where $\operatorname{tr}(\widetilde{L})=3 M_{1} \epsilon^{2} \Gamma_{0}^{2}+M_{2} \eta$ $-\epsilon \partial_{p} \Gamma_{0}$ and $\Delta=-\epsilon \partial_{p} \Gamma_{0}\left(3 M_{1} \epsilon^{2} \Gamma_{0}^{2}+M_{2} \eta\right)-\epsilon \partial_{p} \Gamma_{1}$. The associated eigenvectors $\Psi$ are given by $(2, \operatorname{tr}(\widetilde{L})$

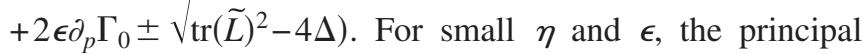
parts of the trace $\operatorname{tr}(\widetilde{L})$, determinant $\Delta$, and discriminant 
$\operatorname{tr}(\widetilde{L})^{2}-4 \Delta$ are given by $M_{2} \eta-\epsilon \partial_{p} \Gamma_{0},-\epsilon \partial_{p} \Gamma_{1}$, and $4 \epsilon \partial_{p} \Gamma_{1}$ respectively. It is clear that the derivatives of $\Gamma_{i}$ at zero points are crucial for stability.

In a view of the profiles of $\Gamma_{i}$ as depicted in Figs. $5(\mathrm{a})-5(\mathrm{c})$, especially the sign of the derivatives at the zero points of $\Gamma_{i}$, stability and bifurcation diagram with respect to the width $d$ at each equilibrium point can be computed as in Figs. 5(d) and 5(e). For the symmetric case $\gamma^{L}=\gamma^{R}$ we have the usual pitchfork bifurcation as shown in Fig. 9 in [45] since there is a reflection symmetry with respect to $p=0$. After introducing the asymmetry, the pitchfork bifurcation points are replaced by their imperfection as shown in Figs. 5(d) and 5(e) with $d$ being the bifurcation parameter and the above reflectional symmetry is lost; nevertheless the bifurcation structure still keeps the symmetry under the transformation $(d, p) \rightarrow(-d,-p)$ except stability properties. We remark here that $\chi(x, 0)$ becomes an odd function when $\gamma_{L} \neq \gamma_{R}$. There are four zero points in $\Gamma_{i}(p, 0)$, thereby the various pulse behaviors besides penetration occur for very small $|d|$ unlike the symmetric case of $\chi(x, 0)=0$ as in [45].

We label each branch as in Figs. 5(d) and 5(e) and thereby LCE is an abbreviation of left-center-equilibrium and similarly for other equilibria. Due to the imperfection, there appear two special branches center-left-equilibrium (CLE) and center-right equilibrium (CRE) that have no bifurcations at all; therefore, for instance, CLE remains as unstable focus for all $d$ when $\epsilon$ is positive and similarly for other cases as depicted in Figs. 5(d) and 5(e). Note that these two fixed points always locate at the steep side of bump, and the remaining part of the imperfection appears via saddle-node bifurcations on the moderate side of bump with labels LCE and right-center equilibrium (RCE). These saddle-node bifurcations around $|d| \approx 0.16$ are associated with the critical case of four zero points of $\Gamma_{i}$ as in Fig. 5(b). Near this critical case, the transition from saddle (gray broken) $\rightarrow$ node (gray dotted) $\rightarrow$ spiral (gray solid) or vice versa is observed along the saddle-node bifurcation branch, although the node regime is very narrow compared with others [not visible in Figs. 5(d) and 5(e)]. This is a consequence of the fact that a real eigenvalue must pass through the origin at the limiting point. Recall that the dominant term of discriminant $\operatorname{tr}(\widetilde{L})^{2}$ $-4 \Delta$ is given by $4 \epsilon \partial_{p} \Gamma_{1}$. The asymmetry of global bifurcation structure is strongly responsible for the onset of unidirectional behaviors as will be discussed in Sec. III B, in which we study the global behaviors of branches with respect to $\epsilon$ including Hopf ones.

\section{B. Bifurcation diagram for bump height $\epsilon$ and onset of rectification behavior}

In this section we study the global bifurcation diagram of ODE system (5) with respect to $\epsilon$ with $d$ being fixed to be 0.10 unless otherwise mentioned. Note that the number of equilibria of Eq. (5) for $d=0.10$ is 3. Figure 8(a) shows the global bifurcation diagram for those equilibria as $\epsilon$ varies. There always occurs Hopf bifurcation along each equilibrium for either positive or negative $\epsilon$ side depending on the sign of $\partial_{p} \Gamma_{0}$. All the Hopf bifurcations are supercritical and hence stable limit cycles are observed at least locally. The black and white circles depict the minimal and maximal positions of limit cycle in $p$ coordinate, which correspond to oscillatory pulses for the original PDE. At first let us consider the positive $\epsilon$ region. The Hopf branch emanating from CLE grows and only the minimal position approaches leftequilibrium (LE) in the form of homoclinic bifurcation at $\epsilon$ $\approx 0.0596$, which is different from the symmetric case $[45,46]$ that the limit cycle grows into the heteroclinc orbit connecting LE and CRE as $\epsilon$ is decreased. It should be noted that a saddle-node bifurcation occurs for this Hopf branch before homoclinic point to LE. The reason is first $\operatorname{tr}(\tilde{L}) \approx M_{2} \eta$ $-\epsilon \partial_{p} \Gamma_{0}$ becomes positive at LE in view of the profile of $\Gamma_{0}$ in Fig. 5, and hence the resulting periodic orbit close to the homoclinic one must be unstable; on the other hand, the Hopf branch is stable near the onset due to its supercriticality. Therefore the Hopf branch behaves globally as depicted in Fig. 8(a). What are the implications of this global behavior? Two important outcomes are derived from this observation: one is a rectification phenomenon and the other is unidirectional pinning-depinning transition. Rectification shows a one-way traffic from right to left, namely, the left-going pulse can penetrate the bump, but right-going one bounces from it. Right after the Hopf bifurcation, the orbit is attracted to the limit cycle as in Fig. 6(a) (upper left). The transition from oscillatory behavior (OSC1) to rebound (REB4) occurs around $\epsilon \approx 0.0607$ when the orbit crosses the stable manifold of LE [dotted line in Fig. 6(a)]. In other words, the stable manifold of LE approaching from right-below direction plays a scattor for this transition. This must occur before the homoclinic bifurcation event since the pulse orbit is not able to access the stable limit cycle at the homoclinic point [see Fig. 6(a)]. On the other hand, it is clear from Fig. 6(a) that the pulse coming from the right must penetrate to $-\infty$, which shows the rectification, namely, only the pulse coming from the right (i.e., steeper side) can go across the bump. As for the pinning-depinning transition, oscillatory trapped pulse corresponds to the stable limit cycle, and this stable one disappears (i.e., depinning) exactly at the saddle-node point of this Hopf branch depicted in Fig. 8(a). The PDE counterpart of LE is numerically confirmed and it should be noted that the similar type of bifurcation occurs for the PDE system as shown in Fig. 7: periodic branch bifurcated from the CLE standing pulse approaches into homoclinic orbit to LE as $\epsilon$ is decreased. To compute the global branches of periodic solutions, we introduce the period $T$ as a time-scaling parameter of the system and let $\Phi$ be the solution of Eq. (1) at time $T$ starting from the initial value $\boldsymbol{u}_{0}$. We set $R=\Phi-\boldsymbol{u}_{0}$ and solve the following linearized system:

$$
\left.\frac{\partial(R, S, N)\left(\boldsymbol{u}, T, k_{1}\right)}{\partial \boldsymbol{u}, T, k_{1}}\right|_{\boldsymbol{u}_{0}, T, k_{1}}\left(\begin{array}{c}
\Delta \boldsymbol{u}_{0} \\
\Delta T \\
\Delta k_{1}
\end{array}\right)=-\left(\begin{array}{c}
R\left(\boldsymbol{u}_{0}, T, k_{1}\right) \\
S\left(\boldsymbol{u}_{0}, T, k_{1}\right) \\
N\left(\boldsymbol{u}_{0}, T, k_{1}\right)
\end{array}\right),
$$

where $S$ and $N$ are the conditions for the phase pinning and pseudoarc length condition under parameter $k_{1}$ variation (see references in [52-54]). Here the problem of finding periodic solutions and their bifurcation branches can be formulated as a periodic boundary value problem of $R=S=N=0$ on a fixed 

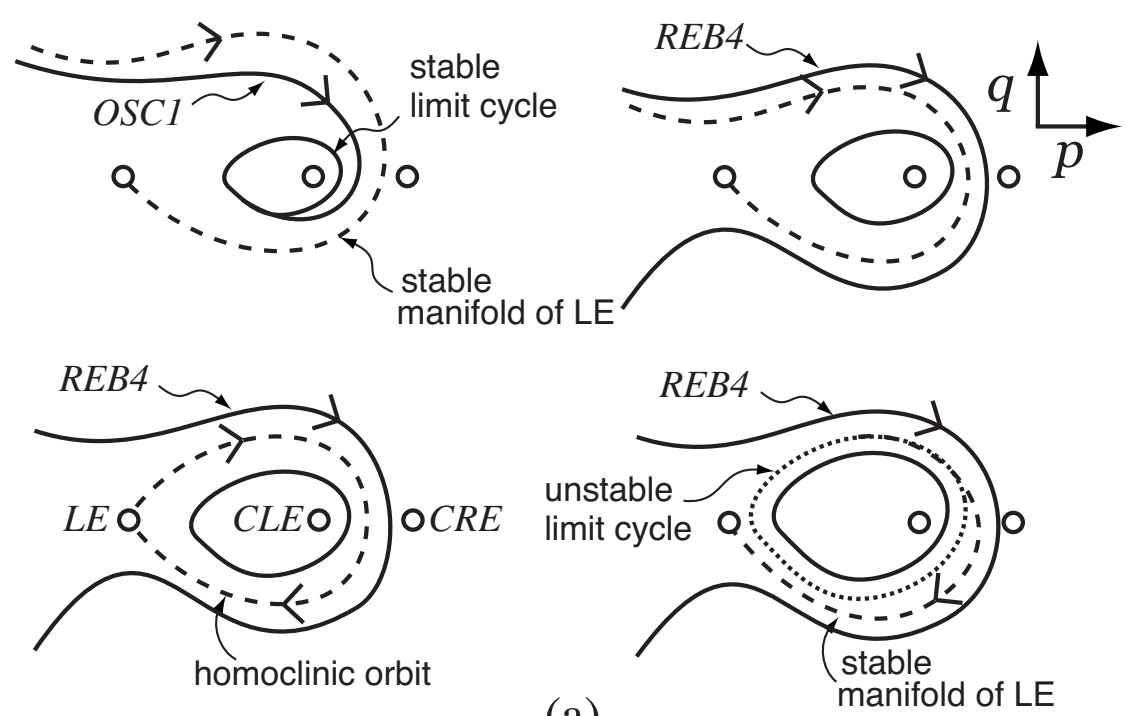

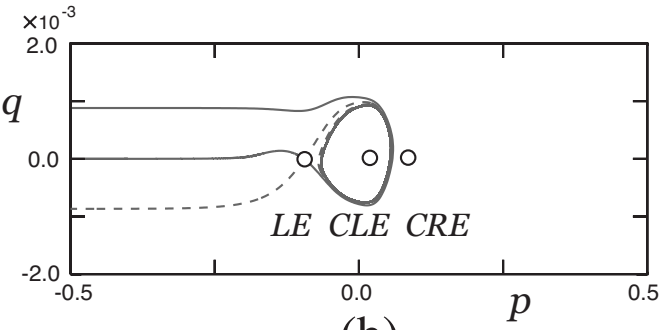

(b)

(a)

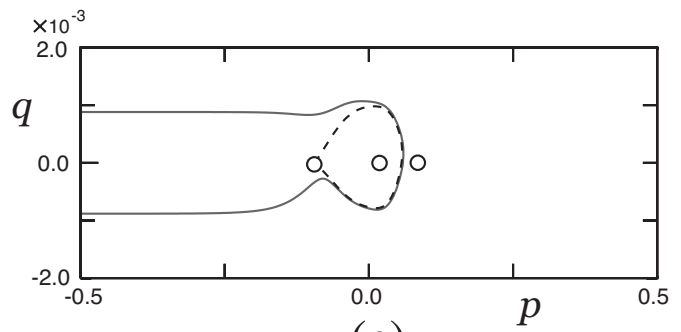

(c)

FIG. 6. (a) The schematic orbit flows near the phase boundary between OSC1 and REB4 near point $B$ from left-top to right-bottom pictures by decreasing $\epsilon$. The gray solid and broken lines indicate the orbit from $E_{2}^{+}(p=-\infty)$ and stable manifold of LE, respectively. The latter plays a role as scattor between OSC1 and REB4 behaviors. As $\epsilon$ is still decreased, the homoclinic orbit to LE appears and its unfolding emanates the unstable limit cycle, which disappears with stable one via a saddle-node bifurcation. The solid and dotted circles indicate stable and unstable limit cycles, respectively. (b) The gray solid and broken lines show the stable and unstable manifolds of LE at $\epsilon \approx 0.0607$, respectively. The homoclinic orbit and the orbit from $E_{2}^{+}(p=-\infty)$ at $\epsilon \approx 0.0596$ are indicated by the black broken and gray solid lines in (c).

period $T$. Unfortunately, a saddle-node point should appear before homoclinic to LE; however the continuation is being stopped before it as $T / \infty$ in Fig. 7(c).
In this way, the asymmetry of our system leads to unidirectional orbit flow to $E_{2}^{-}$of RECT1 resulting in a rebound (REB4) coming from $p=-\infty$ and a penetration coming from

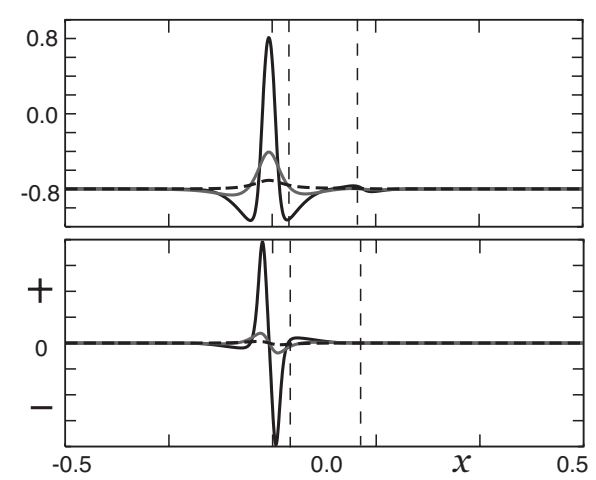

(a)

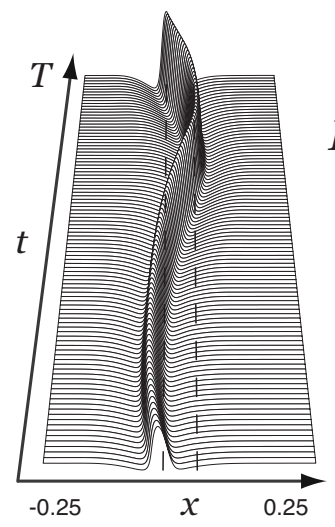

(b)

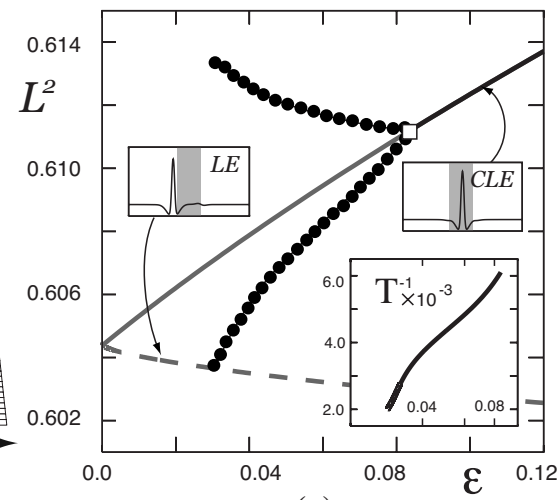

(c)

FIG. 7. (a) The profiles of scattor (top) and the associated eigenfunction (bottom) for $\epsilon \approx 0.0625$ near point $B$ with $\lambda \approx 0.0283$. The solid, gray, and broken lines represent $u, v$, and $w$ components, respectively. (b) Spatiotemporal pattern for $\epsilon \approx 0.0297$ with $T \approx 451$. The periodic orbit spends most of time around the saddle point of LE and approaches into a homoclinic orbit. Global bifurcation branch of limit cycles with respect to the bump height $\epsilon$ for $d=+0.0625$ is shown in (c). The inset figure shows the associated inverse temporal period of limit cycles. 

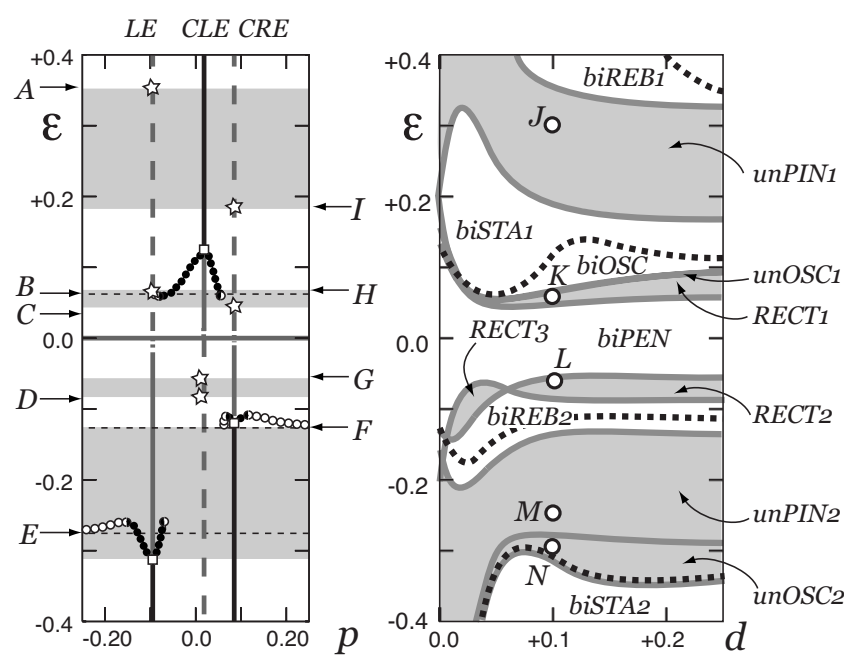

(a)

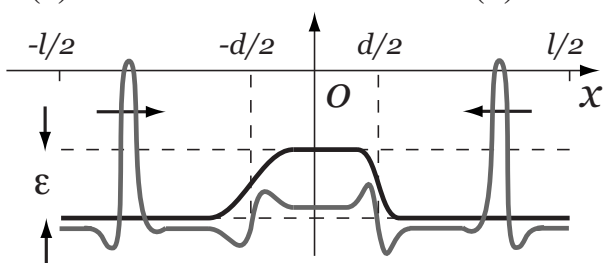

(c)

FIG. 8. Global bifurcation diagram of ODE system with respect to the bump height $\epsilon$ for $d=+0.10$ is shown in (a). The white square indicates the Hopf bifurcation points. The black and white circles indicate the stable and unstable limit cycles, respectively. The horizontal broken lines indicate the homoclinic bifurcations for the limit cycles. The black and gray solid lines indicate the stable and unstable foci, respectively, and the dark gray broken line indicates the unstable saddle. The additional characters $(A-I)$ correspond to those marked on the phase boundaries in Fig. 2(a). The five-pointed stars indicate the scattors observed at the phase boundaries. (b) Phase diagram for the unidirectional behaviors in the presence of an asymmetric heterogeneity. Right-going (left-going) pulse hits first moderate (steep) side of bump as depicted schematically in (c). There are seven different unidirectional behaviors indicated by gray color. The dotted lines correspond to the Hopf bifurcation lines for the critical points.

$p=+\infty$ as shown in Fig. 9(c). The same thing happens in PDE dynamics as in Fig. 1(b). It looks trivial that, when $\epsilon$ is still decreased, pulses can go across the heterogeneity in bidirectional manner. Let us look carefully at the behaviors near the transition to bidirectional penetration (biPEN). In view of Fig. 4(c), the transition from REB4 to PEN coming from $E_{2}^{+}(p=-\infty)$ is responsible for that from RECT1 to biPEN, i.e., the stable manifold of CRE point plays a role as separator among them.

\section{Unidirectional flows and scattors}

The bidirectional and unidirectional behaviors are classified as in the phase diagram of Fig. 8(b), depending on two parameters $(d, \epsilon)$. There are six different bidirectional outputs-bipenetration (biPEN), birebound (biREB1, biREB2), and bistationary (biSTA1, biSTA2), and bioscillatory
(biOSC) states - as well as seven unidirectional outputsrectification (RECT1, RECT2, RECT3), unidirectional pinning (unPIN1, unPIN2), and unidirectional oscillatory (unOSC1, unOSC2) states. Note that the diagram of Fig. 8(b) can also be produced as the superposition of the diagram of Fig. 2(a) and its $\pi$ rotation. According to Remark II.1, our system is invariant under the transformations $(d, \epsilon) \rightarrow(-d$, $-\epsilon)$, so it suffices to consider the positive $d$ space as in Fig. 8(b). We indicate the phases of unidirectional behaviors in gray and the bidirectional behaviors in white. The typical unidirectional behaviors for $d=0.10$ are depicted in Fig. 9. In this section, we focus on the nature of dynamic transition among these states captured in the bifurcation diagram of Fig. 8(a).

As it is expected, the biPEN behavior occurs when $|\epsilon|$ is small, i.e., pulses can go across the bump heterogeneity. By increasing $\epsilon$, the rectification behavior RECT1 of Fig. 9(c) appears for small $\epsilon$ as described in Sec. III B. As $\epsilon$ is still increased, the unOSC 1 region appears, which is very narrow compared with the others [not visible in Fig. 8(b)]. Similar dynamics is observed for the PDE dynamics as in Fig. 1(b). The stable manifolds of LE and CRE play a role as scattors for the transitions from biPEN and to biOSC, those are indicated as the five-pointed stars at $B$ and $C$ in Fig. 8(a), respectively. The unOSC1 dynamics is shown in Fig. 9(b), where the right-going pulse could be trapped by the oscillatory state around CLE. Due to the type change in global bifurcation from heteroclinic to homoclinic, the two unidirectional behaviors emerge between the bidirectional behaviors of biPEN and biOSC. In particular, the $(d, \epsilon)$ region where RECT1 behaviors are observed coincides with the REB4 region in Fig. 2(a).

When $\epsilon$ changes its sign, there is an another rectification behavior of RECT2 which direction is reversed to $E_{2}^{+}(+\infty)$, left-going pulses rebound at the flip dent of case II, however right-going pulses could penetrate the dent of case IV. Figure 9(d) shows the corresponding behavior near point $L$ in Fig. 8(b). The PDE counterpart has already been depicted in Fig. 1(c).

The asymmetry of the system results in the symmetry breaking of critical point distribution as well as the geometry of their manifolds. The phase boundaries of RECT2 region are dominated by the scattors associated with those obtained near points $D$ and $G$ as indicated in Fig. 8(a). The scattor of transition from biREB2 to RECT2 is the upper-left stable manifold of LE, which is the same as that obtained at the transition from PEN to REB2 at point $D$ in view of Fig. 3(d). As $\epsilon$ is still decreased, this stable manifold is also responsible for the transition from RECT2 to biPEN, which is associated with that of CRE at point $G$ as indicated in Fig. 4(b) for case III. Here the CRE in case III could be replaced into LCE in case I under the transformations $(d, p) \rightarrow(-d,-p)$. Therefore the stable manifold of CLE in case I is the scattors for both transitions as shown in Fig. 10(a) (A). The position of middle fixed point glides right due to the imperfection of the bifurcation structure of Fig. 5, and then there occurs a gap of the $\epsilon$ value where the orbit from the left (right) end meets the upper-left (lower-right) stable manifold of CLE. When the system recovers the symmetry, the situation changes dramatically because the CLE scattors are merged 


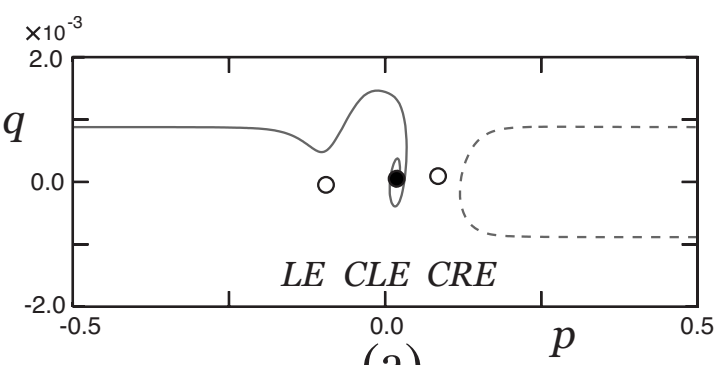

(a)

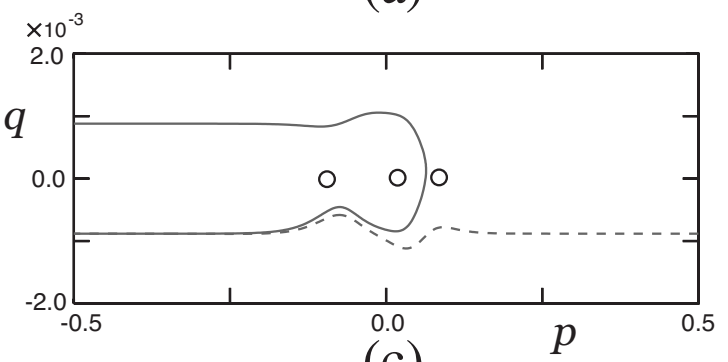

(c)

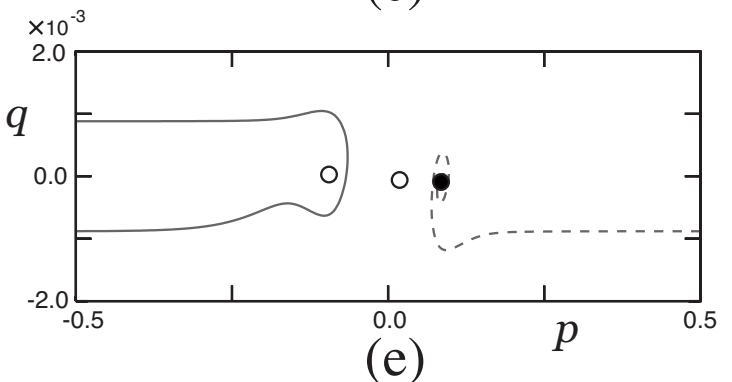

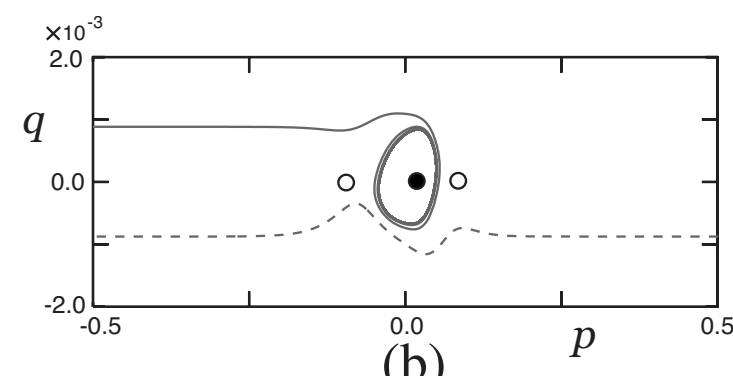

(b)

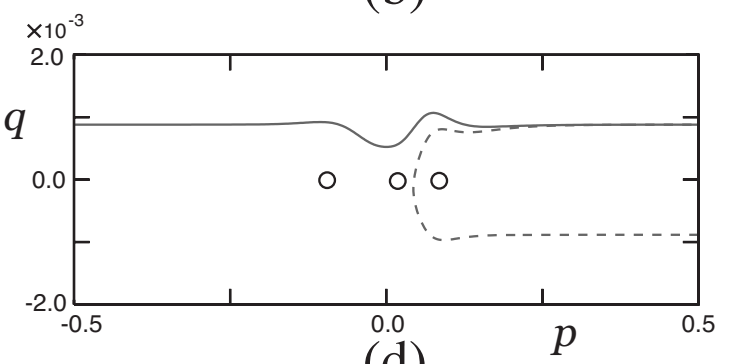

(d)

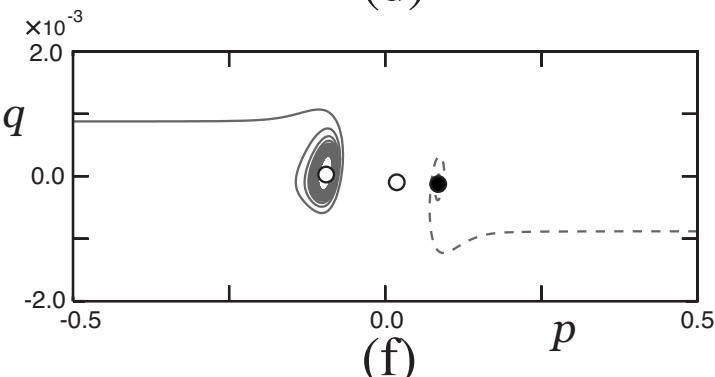

FIG. 9. Unidirectional ODE flows in the presence of asymmetric heterogeneity are associated with the PDE behaviors shown in Fig. 1. The solid and broken lines indicate the orbit flows starting from $E_{2}^{ \pm}$at $p=\mp \infty$, respectively. The unidirectional pinning behaviors of (a) unPIN1consist of STA1 from the left and REB2 from the right occurs around $(d, \epsilon)=(0.10,0.30)$ near point $J$ in Fig. 8(b), (b) unOSC1 of OCS1 and PEN around $(d, \epsilon)=(0.10,0.07)$ near point $K$. The two rectification behaviors are obtained as (c) RECT1 consists of REB4 from the left and PEN from the right around $(d, \epsilon)=(0.10,0.055)$ near point $K$ and (d) RECT2 of PEN and REB1 for $(d, \epsilon)=(0.10,-0.055)$ near point $L$. There are the other unidirectional pinning behaviors for negative $\epsilon$, (e) unPIN2 consists of REB2 from the left and STA1 from the right around $(d, \epsilon)=(0.10,-0.25)$ near point $M$. (f) unOSC2 of OCS3 and STA1 around $(d, \epsilon)=(0.10,-0.30)$ near point $N$. The solid and open circles indicate the stable and unstable stationary states, respectively.

into one point of center-equilibrium (CE) as in Fig. 10(a) (B) and the unidirectional behavior of RECT2 disappears. In this way, the rectification phases are observed for a window of small $\epsilon$ values until a critical value of $\epsilon$ is reached resulting in bidirectional pulse penetration, as $|\epsilon|$ is decreased. Only with the inversion of the propagating direction, the same thing happens to RECT3 region for small $d$. Note that transition from biREB2 to biPEN occurs at a connecting point of RECT2 and RECT3 regions, in which the stable manifolds of CLE play a role as scattors simultaneously.

For large positive $\epsilon$, the unidirectional pinning behavior of unPIN1 is observed, where the left-going pulse coming from $p=+\infty$ rebounds from the flip bump of case III and the right-going pulse coming from $p=-\infty$ could be trapped by the pinning state of STA1 around the steep side of bump. The overall dynamics is shown in Fig. 9(a). This unidirectional flow is also caused by the positional change between the scattors and the orbits starting from both ends under symmetry breaking of the system. The fates of unPIN1 orbit flows are sorted out along the unstable manifolds of CRE at points $A$ and $I$ in Fig. 8(a). Since the stable manifold of CRE is a basin boundary, any trajectory starting from $p=+\infty$ leads to rebound from it. Hence, the basin of attraction for stable CLE is not accessible from the steep side of bump.

The other unidirectional pinning behaviors of unOSC2 and unPIN2 are due to the positional relation of Hopf and homoclinic bifurcation points for negative $\epsilon$ in Fig. 8(a), i.e., the global branches of periodic solutions play a key role in understanding the onset of these behaviors. There are two supercritical Hopf bifurcations from below. The coexistence of the different slope influences the Hopf point locations among both side critical points. In view of the profiles of the heterogeneous functions $\Gamma_{i}$ in Fig. 5(a), since $\operatorname{tr}(\tilde{L}) \approx M_{2} \eta$ $-\epsilon \partial_{p} \Gamma_{0}=0$ and $\operatorname{tr}(\tilde{L})^{2}-4 \Delta \approx 4 \epsilon \Gamma_{1}<0$, the Hopf point of the moderate side LE is smaller than that for steep side CRE. Thus, the LE critical point at moderate side first undergoes a Hopf bifurcation around $\epsilon \approx-0.315$ that produces pinning behavior to oscillatory motion. Figure 9(f) shows the unOSC2 behavior near point $N$ in Fig. 8(b), where one describes the trajectory of oscillatory trapping around LE and the other describes the trajectory to stationary pinning to CRE. Similar dynamics is also observed for PDE dynamics as depicted in Figs. 1(d) and 1(e). As $\epsilon$ is still increased, the amplitude of this limit cycle increases leading to a ho- 


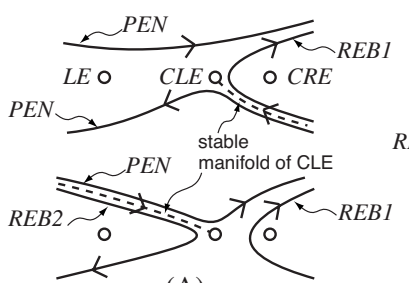

(A)

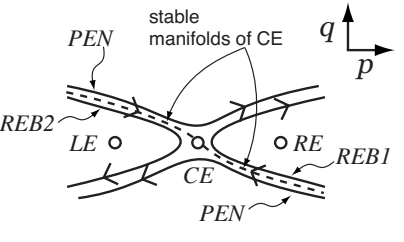

(B)

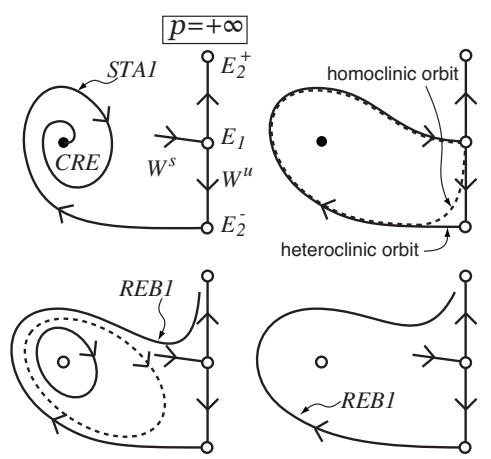

(b)

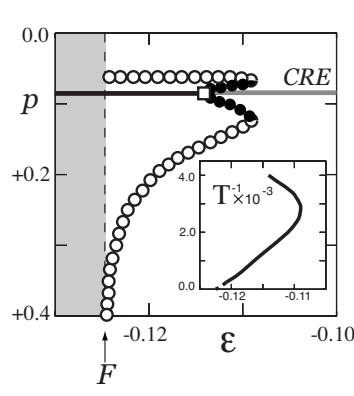

(c)
FIG. 10. (a) The schematic orbit flows explain how the asymmetric critical point distribution changes the orbital relations to scattors and causes the RECT2 behaviors between bidirectional behaviors as in (A). As $\epsilon$ is decreased from top to bottom pictures; there appear two scattors located at CLE point for transitions from biPEN to RECT2 and from RECT2 to biREB, respectively. They correspond to the scattors obtained near points $D$ and $G$ in Fig. 8(a), respectively. When the system recovers the symmetry, they are merged into one scattor like (B) and the rectification behavior disappears. (b) The schematic orbit flows near the phase boundary between unPIN2 and biREB2 from left-top to right-bottom pictures, corresponding to the homoclinic bifurcation near point $F$ in Fig. 8(a). A homoclinic orbit to saddle $E_{1}$ belongs to the intersection of unstable and stable manifolds of $E_{1}$. An unstable limit cycle (dotted line) appears from the homoclinic bifurcation and it disappears with the stable one by saddle-node bifurcation, as $\epsilon$ is increased. The black and white circles indicate the stable and unstable equilibrium points, respectively. (c) Global behavior of periodic branch around CRE point is magnified near point $F$ in Fig. 8(a). The limiting point of the limit cycle locates at $\epsilon \approx-0.109$. The inset figure shows the associated inverse temporal period of limit cycles.

moclinic orbit via a saddle-node bifurcation at $\epsilon \approx-0.274$. This bifurcation at point $E$ in Fig. 8(a) generates the rebound behavior of pulses coming from $E_{2}^{+}(-\infty)$ and then leads to transition from unOSC2 to unPIN2.

Another homoclinic bifurcation of limit cycle at the steep side is responsible for the transition from unPIN2 to biREB2 behaviors. The limit cycle emanated from the Hopf point of CRE around -0.114 grows and terminates in homoclinic bifurcations via saddle-node points at $\epsilon \approx-0.124$. The schematic orbital behaviors near the transition are depicted in Fig. 10(b). Near point $F$ in Fig. 8(a), the trajectory coming from $E_{2}^{-}(+\infty)$ rebounds from the flip dent of case II, in which unstable periodic branch blocks the entering of the orbit into the inside attractors. Qualitatively similar dynamics has been discussed in Fig. 12(c) in [45]. As $\epsilon$ is still increased, therefore, the unPIN2 region terminates in a homoclinic bifurcation at the steep side.
The reduced ODE dynamics shown in Fig. 9 almost completely describes the PDE behavior depicted in Fig. 1. A remarkable feature is that the types of unidirectional behaviors are not only uniquely determined by the shape of the asymmetry (i.e., by the fact which side of the slope is steeper) but also by the height and the width of the bump heterogeneity, in which we discussed here three types of the onset of the unidirectional behaviors from the global bifurcation picture. The first is due to the asymmetric distributions of critical points, i.e., the imperfection of the bifurcation structure changes the orbital relations to the associated scattors. The second is that the change from heteroclinic to homoclinic bifurcation is crucial to the onset of the rectification behavior; in fact, there occurs two unidirectional behaviors between bidirectional penetration and pinning behaviors. Third, the global behaviors of oscillatory branches play a key role in understanding the unidirectional pinning behaviors, depending on the positional order of local and global bifurcation points. It should be remarked that the propagation direction accessible to pinning state as well as rectification could be reversed by changing the sign of $\epsilon$.

\section{CONCLUSION}

We have studied the onset of unidirectional behaviors of pulses as well as pinning-depinning transition in a medium with an asymmetric heterogeneity. When such a heterogeneity is introduced to the model system of Eq. (1) in an additive way, heterogeneity-induced standing pulses (called defects) are created near the edge of the obstacle. The dynamics of pulses near the heterogeneity is governed by the properties of the collision process between pulses and defects. In fact, a variety of outputs (see Figs. 1 and 2) are observed as a consequence of qualitatively different collision events with defects depending on the height and width of the bump. The methodology developed in $[45,46]$ for collision problems can be therefore applied to our problem.

In particular the reduction from the original PDEs to a system of ODEs allows us to study the global bifurcation structure of the problem. Thereby, we could not only clarify the existence and stabilities of defects induced by the presence of the heterogeneity but also demonstrate the impact of the asymmetry on the bifurcation diagram, which is rendered imperfect due to symmetry-breaking terms. It turns out that such a global bifurcation structure is crucial for understanding the onset of unidirectional behaviors.

We have examined the two types of unidirectional behaviors and identified the underlying mechanism whereby they take place. The first is the Hopf bifurcations of defects. The emerging limit cycles grow in amplitude and finally collide with saddle points at homoclinic global bifurcation points. The outcome of the interaction of pulses with the heterogeneities is dominated by the asymmetric distribution of scattors and the positional relation between the local Hopf and the global bifurcation point. The second aspect is the switching of connectivity from heteroclinic to homoclinic type due to the asymmetry, which is responsible for the appearance of the rectification behavior schematically depicted in Fig. 11. Figure 12 shows multipulse-rectification behaviors for the 


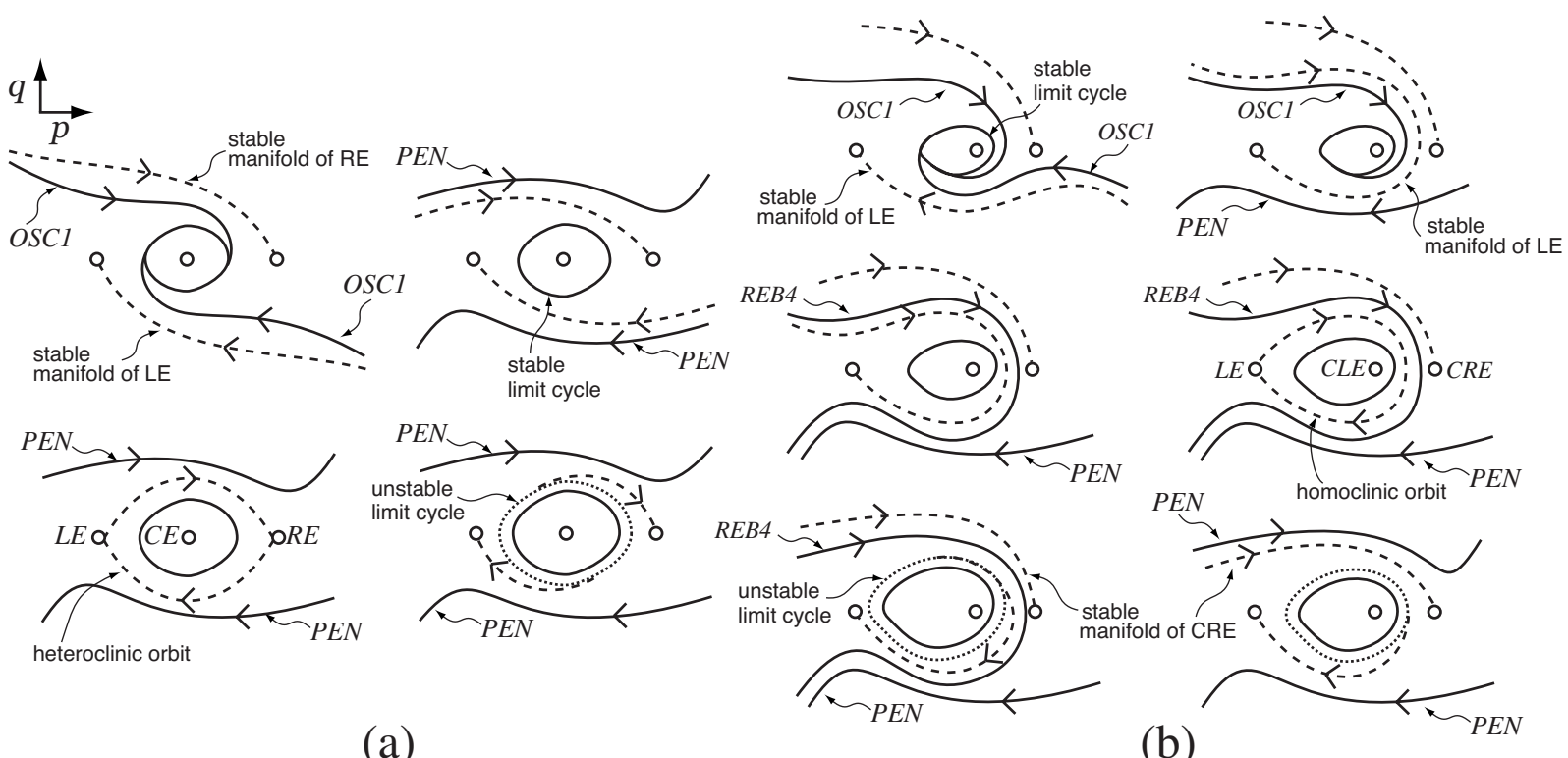

FIG. 11. The schematic orbit flows explain how the change of type of global bifurcation causes the RECT1 and unOSC1 behaviors between bidirectional behaviors for positive $\epsilon$ region. (a) For the symmetric case, bidirectional oscillatory pinning (biOSC) behavior changes into biPEN behavior via a double heteroclinic bifurcation from left-top to right-bottom pictures by decreasing $\epsilon$. (b) The heterogeneous media are changed from symmetric to asymmetric; double heteroclinic loop is transformed into homoclinic one. The unidirectional oscillatory pinning behavior (unOSC1) and the rectification to the left (RECT1) behaviors occur between bidirectional behaviors as $\epsilon$ is decreased from left-top to right-bottom pictures. The lower-right stable manifold of LE plays a role of scattors at transitions from biOSC to unOSC1 and from unOSC1 to RECT1, respectively. As $\epsilon$ is still decreased, the upper-left stable manifold of CRE becomes the scattor at transition between RECT1 and biPEN [see Fig. 6(a)]. The solid (broken) lines indicate the orbit flows from $E_{2}^{ \pm}(p=\mp \infty)$ (stable manifold of critical points). The solid (dotted) circles show the stable (unstable) limit cycles and the white circle indicates the unstable critical points.

special case of a periodic heterogeneity consisting of four bumps. Each domain profile is given by Eq. (2). When the height is $\epsilon=0.06$, all pulses are finally rectified and travel to the left. On the other hand, the final propagation direction is switched by changing the sign of $\epsilon$ [see Fig. 12(b)] and the direction of rectification is inverted. The final form of ODEs does not depend on the details of the model system, i.e., the information specific to the form and parameters of the PDE is contained in the coefficients and the form of the heterogeneity functions $\Gamma_{i}$. The approach presented here has a great potential for the application to a wider class of reactiondiffusion systems and may be employed in the design and control of new experiments.

Finally, we consider the implications of the codimension 2 bifurcation theory. We found the saddle-node structure formed by left-left-center equilibrium (LLCE) and LCE branches with respect to $d$ as in Fig. 5. Recalling the Hopf bifurcations, this implies that there may exist the BogdanovTakens (BT) points at which saddle-node and Hopf bifurcations occur simultaneously. According to the bifurcation condition of $\operatorname{tr}(\widetilde{L})=\Delta=0$, such points locate at quite large $\epsilon$. This indicates that the unfolding of the BT bifurcation may not be a reasonable candidate for the organizing center for the whole bifurcation picture. However, the other codimension 2 bifurcation can certainly be expected to occur for the symmetric case of $\gamma_{L}=\gamma_{R}$, where we have already seen the important role played by the figure-of-eight bifurcations $[45,46]$. In Appendix, we discuss the imperfection of figureof-eight bifurcation in more detail.

\section{ACKNOWLEDGMENTS}

This work was partially supported by the Japanese Ministry of Education, Science, Sports and Culture, Grant-in-Aid for Scientific Research (A) under Grant No. 16204008 and the Japanese Ministry of Education, Science, Sports and Culture, Grant-in-Aid for Young Scientists (B), under Grant No. 20740224. Financial support by Sonderforschungsbereich/

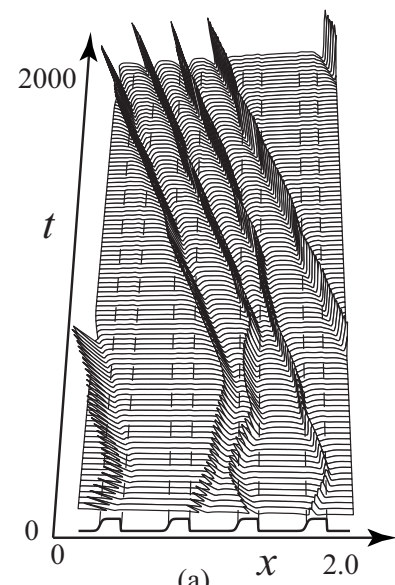

(a)

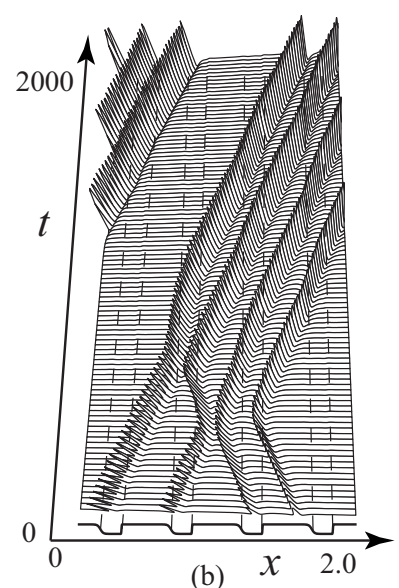

(b)
FIG. 12. Control of multipulses rectification behaviors by using periodic heterogeneity. (a) Four pulses are rectified to the left direction by four bump domains for $\epsilon=0.06$. (b) The propagation direction is reversed to the right by changing the sign of $\epsilon$ as $\epsilon=-0.04$. The other parameters are the same as those used in Fig. 1. 


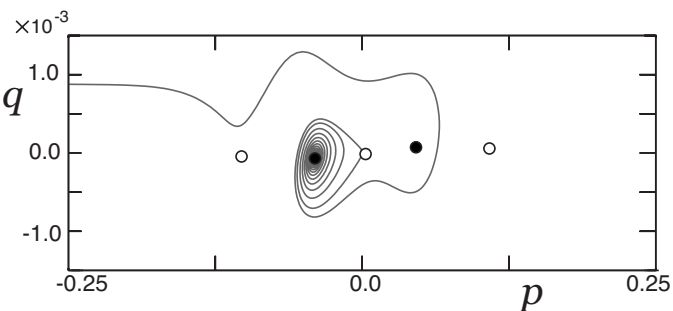

(a)
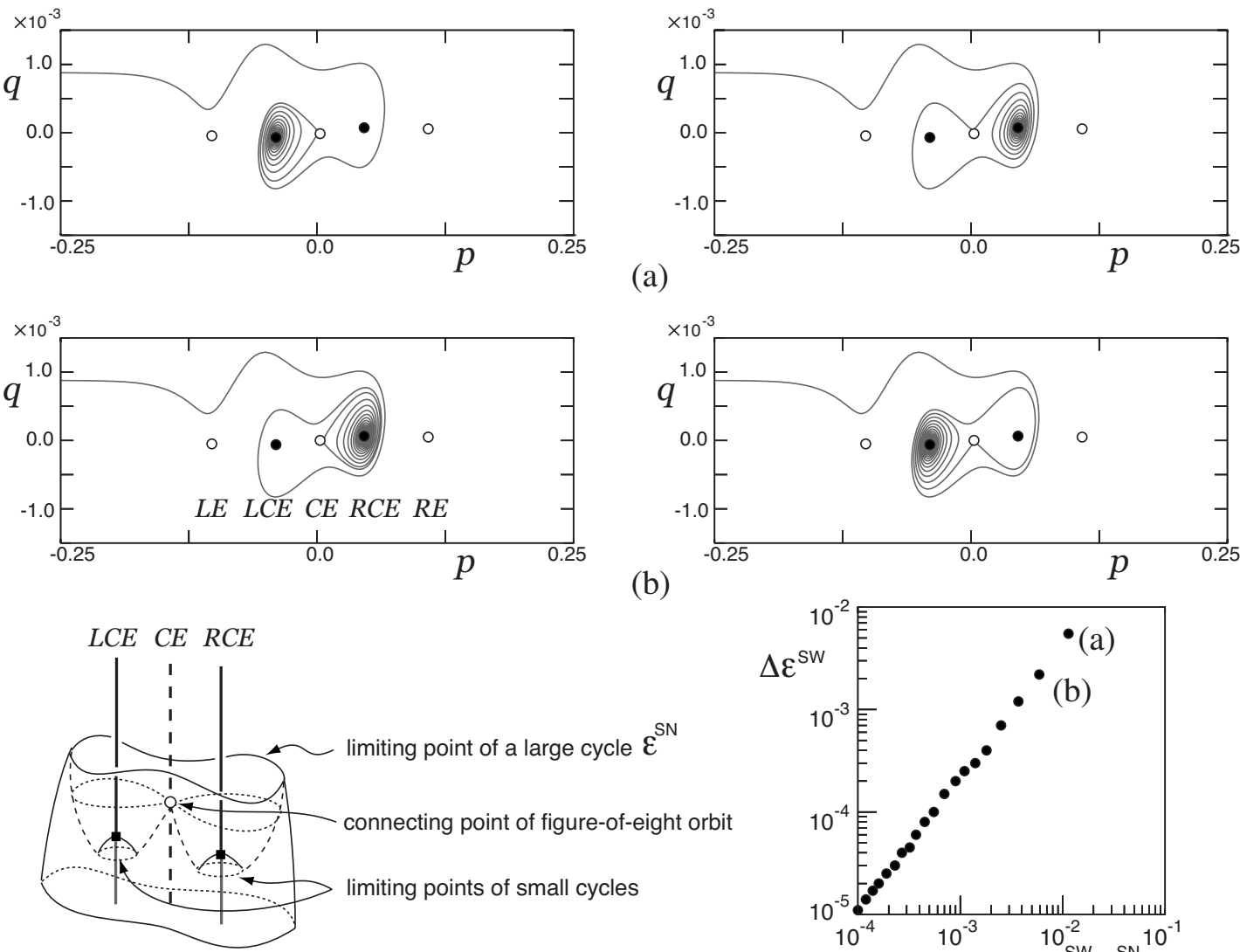

(c)

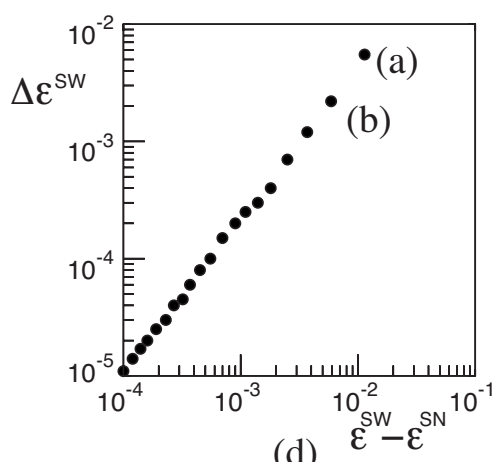

(d)

FIG. 13. Attractor switching phenomenon and figure-of-eight bifurcation for the wide case of the symmetric steep bump of $\left(\gamma^{L}, \gamma^{R}\right)$ $=(100,100)$. (a) Orbit flows of STA3 near the attractor switching point at $\epsilon \approx 0.156$. Slightly decreasing $\epsilon$, the orbits are sorted out from LCE to RCE by the upper-left unstable manifold of CE. (b) The attractor is switched from RCE to LCE by the lower-right unstable manifold of $\mathrm{CE}$ at $\epsilon \approx 0.151$. The whole structure of the limit cycles for positive $\epsilon$ forms a surface similar to trousers. Small limit cycles around LCE and $\mathrm{RCE}$ are emanated from the supercritical Hopf bifurcations at $\epsilon \approx 1.32$. These two cycles merge into one large cycle via a figure-of-eight bifurcation at $\epsilon \approx 0.142$. The connected limit cycle disappears by the heteroclinic bifurcations at $\epsilon \approx 0.040$. All three cycles change the stability via saddle-node bifurcations at $\epsilon \approx 0.145$ and 0.131 , respectively. (d) Log-log plot of the $\epsilon$ values for the switching points. The horizontal axis is $\epsilon^{\mathrm{sw}}-\epsilon^{\mathrm{sn}}$ and the vertical axis is $\Delta \epsilon^{\mathrm{sw}}$. There occurs an infinite number of the attractor switching approaching $\epsilon^{\mathrm{sw}}$ into $\epsilon^{\mathrm{sn}}$. The orbital behaviors near the first two switching points marked on the figure correspond to (a) and (b).

Collaborative Research Center "Complex Nonlinear Processes" (Grant No. SFB 555) of Deutsche Forschungsgemeinschaft (DFG) is gratefully acknowledged.

\section{APPENDIX: IMPERFECTION OF FIGURE-OF-EIGHT BIFURCATION}

We have considered here so far only the narrow asymmetric bump, for which the heterogeneity function $\Gamma_{1}$ has three zeroes. In our previous study for symmetric wide bump, in which five zero points are saddles and foci alternatively, we have sketched the unfolding of figure-of-eight (double homoclinic, ambiclinic) bifurcation of limit cycles and the associated characteristic behaviors of STA3 for steep slope case or OSC3 for moderate slope case were obtained (see references in $[45,46])$. Here the STA3 phase behavior for the steep wide bump in [46] can be traced further. As $\epsilon$ is decreased, the orbit approaches the upper stable manifold of LE and it encounters transition from REB1 to STA3. As $\epsilon$ is still decreased, the orbital behavior changes from STA3 to
OSC1 via a saddle-node bifurcation for the large limit cycle.

As depicted in Fig. 13(c), no limit cycles are left above the limiting point at $\epsilon^{\mathrm{sn}} \approx 0.145$. Two stable foci of LCE and RCE can be expected to be attractors of STA3. Actually, there occurs attractor switching phenomenon between LCE and RCE. It is verified numerically that as shown in Fig. 13(a), the orbit from the left stays very close to CE saddle point at $\epsilon \approx 0.156$, then it is sorted along the direction of the unstable manifold, giving rise to attractor switching from LCE to RCE by decreasing $\epsilon$. It switches again at $\epsilon$ $\approx 0.151$ [see Fig. 13(b)], converging to LCE that switches into RCE at $\epsilon \approx 0.149 \cdots$. The fate of the orbit from the right is also switched between LCE and RCE alternatively.

The important point is that the attractor switchings between LCE and RCE are controlled by the upper and lower stable manifolds of CE alternatively, approaching in the limiting point of $\epsilon^{\mathrm{sn}}$. To illustrate this behavior, as shown in Fig. 13(d), it is useful to investigate the dependence on $\epsilon^{\mathrm{sw}}-\epsilon^{\mathrm{sn}}$ of $\Delta \epsilon^{\mathrm{sw}}$. Notation $\Delta \epsilon^{\mathrm{sw}}$ is the difference between successive switching points. As $\epsilon^{\mathrm{sw}}$ approaches $\epsilon^{\mathrm{sn}}$, converging to LCE 


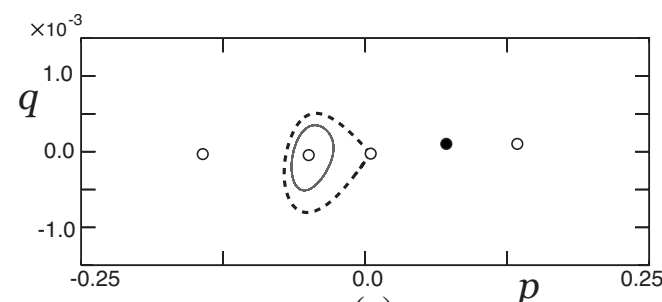

(a)

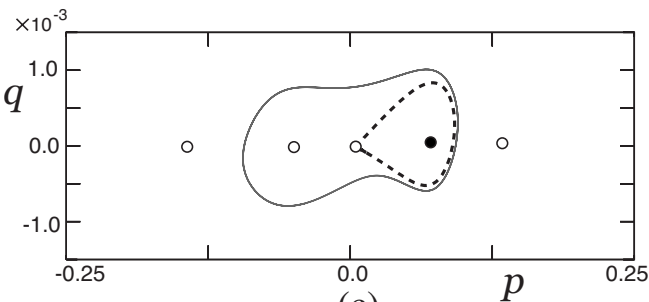

(c)

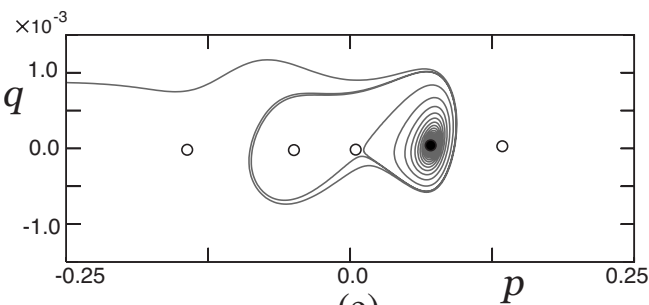

(e)

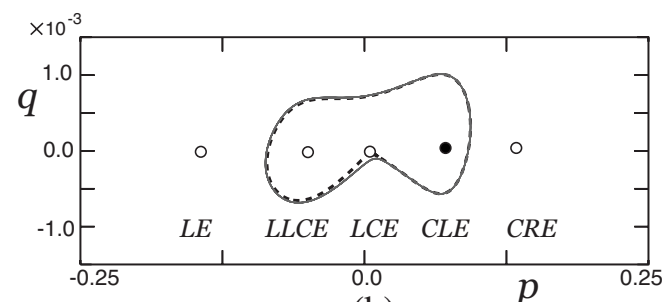

(b)

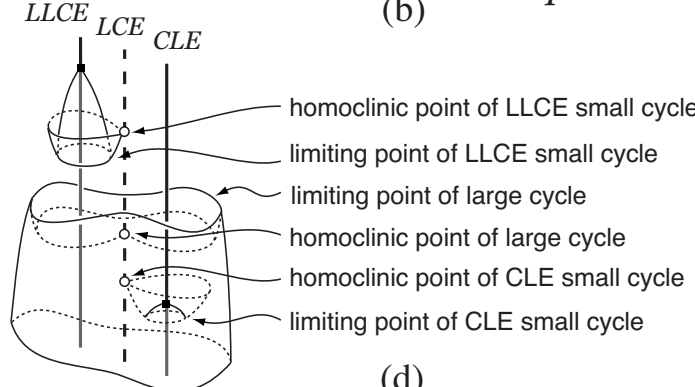

(d)

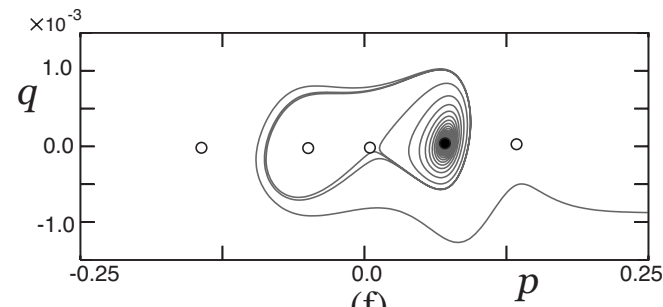

(f)

FIG. 14. Imperfection of figure-of-eight bifurcation for the wide case of $d=0.20$ for the asymmetric bump of $\left(\gamma^{L}, \gamma^{R}\right)=(50,100)$. (a) The gray solid and black broken lines show the stable small limit cycle and homoclinic orbit to LLCE at $\epsilon \approx 0.364$. (b) The gray solid and black broken lines show the stable large limit cycle and homoclinic orbit to LCE at $\epsilon \approx 0.1343$. (c) The gray solid and black broken lines show the stable large limit cycle and homoclinic orbit to LCE at $\epsilon \approx 0.122$. The solid and open circles indicate the stable and unstable stationary states, respectively. (d) The whole structure of the limit cycles with respect to $\epsilon$. Small limit cycles around LLCE and CLE points are emanated from the supercritical Hopf bifurcations at $\epsilon \approx 0.400$ and 0.125 , respectively. They lose the stability via saddle-node bifurcations at $\epsilon$ $\approx 0.353$ and 0.115 , respectively. The limiting point of a large limit cycle locates at $\epsilon \approx 0.1344$ and it disappears by the homoclinic bifurcation of LCE point at $\epsilon \approx 0.1343$. [(e) and (f)] Orbit flows of biSTA1 at $\epsilon \approx 0.1345$ which is close to a saddle-node bifurcation for the large limit cycle. There remains a temporary large oscillatory motion and the orbit will converge to the stable focus of LCE.

or RCE, an infinite number of attractor switching occurs, i.e., an orbit pass nearby CE iteratively, and the differences $\Delta \epsilon^{\mathrm{sw}}$ are getting thinner and thinner. The scaling law of $\Delta \epsilon^{\mathrm{sw}}$ $\approx \kappa\left(\epsilon^{\mathrm{sw}}-\epsilon^{\mathrm{sn}}\right)^{3 / 2}$ suggests that the saddle-node bifurcation of limit cycles is crucial to the attractor switching phenomenon, in which the number of iterates can be estimated as $N\left(\epsilon^{\mathrm{sw}}\right)$ $\approx(2 / \kappa)\left(\epsilon^{\mathrm{sw}}-\epsilon^{\mathrm{sn}}\right)^{-1 / 2}$, where $\kappa$ is a constant [23,24,55].

For the asymmetric case with $\gamma^{L} \neq \gamma^{R}$, the breakdown of figure-of-eight orbit splits into a series of three homoclinic orbits. Two foci of LLCE and CLE are stable for large $\epsilon$. As $\epsilon$ is decreased, the supercritical Hopf bifurcations lead to the appearance of two small limit cycles. They lose stability via saddle-node bifurcations and then grow and collide with the LCE saddle branch via homoclinic bifurcations as shown in Fig. 14(d). A large limit cycle surrounding the three critical points emerges at another homoclinic point with $\epsilon \approx 0.1343$.
All branching limit cycles connecting to each homoclinic points become unstable since $\partial_{p} \Gamma_{0}$ at LCE is negative, i.e., $\operatorname{tr}(\tilde{L})>0$. The three homoclinic orbits are numerically confirmed as in Figs. 14(a)-14(c). As seen from the figures of Figs. 13(c) and 14(d), the question of whether or not imperfection is allowed which destroys a figure-of-eight bifurcation must be addressed in bifurcation problems.

Figures 14(e) and 14(f) show the orbit flows starting from $E_{2}^{\mp}$ at $p= \pm \infty$, in which the attractor switching phenomenon disappears due to the imperfection of figure-of-eight bifurcation. Above the limiting point of a large cycle, the orbits can approach into the LCE point only from the direction of rightlower stable manifold; therefore they must be directed to the CLE point. As a result, by increasing $\epsilon$, transition from biOSC to biSTA1 (to only CLE) occurs via a saddle-node bifurcation of large limit cycle. 
[1] M. Bär, M. Eiswirth, H.-H. Rotermund, and G. Ertl, Phys. Rev. Lett. 69, 945 (1992).

[2] M. Bode, A. W. Liehr, C. P. Schenk, and H.-G. Purwins, Physica D 161, 45 (2002).

[3] Y. Hayase and T. Ohta, Phys. Rev. E 62, 5998 (2000).

[4] C. P. Schenk, M. Or-Guil, M. Bode, and H.-G. Purwins, Phys. Rev. Lett. 78, 3781 (1997).

[5] V. K. Vanag and I. R. Epstein, Chaos 17, 037110 (2007).

[6] M. Bär, E. Meron, and C. Utzny, Chaos 12, 204 (2002).

[7] M. Bode, Physica D 106, 270 (1997).

[8] P. Schütz, M. Bode, and H. G. Purwins, Physica D 82, 270 (1995).

[9] M. Bär, I. G. Kevrekidis, H.-H. Rotermund, and G. Ertl, Phys. Rev. E 52, R5739 (1995).

[10] G. B. Ermentrout and J. Rinzel, SIAM J. Appl. Math. 56, 1107 (1996).

[11] M. Gutman, I. Aviram, and A. Rabinovitch, Phys. Rev. E 69, 016211 (2004).

[12] A. Hagberg, E. Meron, I. Rubinstein, and B. Zaltzman, Phys. Rev. Lett. 76, 427 (1996).

[13] A. Hagberg, E. Meron, I. Rubinstein, and B. Zaltzman, Phys. Rev. E 55, 4450 (1997).

[14] T. J. Lewis and J. P. Keener, SIAM J. Appl. Math. 61, 293 (2000).

[15] J. Miyazaki and S. Kinoshita, Phys. Rev. E 76, 066201 (2007).

[16] A. Prat, Y.-X. Li, and P. Bressloff, Physica D 202, 177 (2005).

[17] J. Xin, SIAM Rev. 42, 161 (2000).

[18] A. M. Pertsov, E. A. Ermakova, and E. E. Shnol, Physica D 44, 178 (1990).

[19] A. M. Zhabotinsky, M. D. Eager, and I. R. Epstein, Phys. Rev. Lett. 71, 1526 (1993).

[20] G. Haas, M. Bär, I. G. Kevrekidis, P. B. Rasmussen, H.-H. Rotermund, and G. Ertl, Phys. Rev. Lett. 75, 3560 (1995).

[21] N. Hartmann, Y. Kevrekidis, and R. Imbih, J. Chem. Phys. 112, 6795 (2000).

[22] P. Schütz and M. Bode, Int. J. Bifurcation Chaos Appl. Sci. Eng. 6, 1891 (1996).

[23] G. Dangelmayr, J. Hettel, and E. Knobloch, Nonlinearity 10, 1093 (1997).

[24] E. Knobloch, J. Hettel, and G. Dangelmayr, Phys. Rev. Lett. 74, 4839 (1995).

[25] A. Adamatzky, B. D. L. Costello, and T. Asai, ReactionDiffusion Computers (Elsevier, New York, 2005).

[26] L. Kuhnert, K. I. Agladze, and V. I. Krinsky, Nature (London) 337, 244 (1989).

[27] A. S. Mikhailov and K. Showalter, Phys. Rep. 425, 79 (2006).

[28] O. Steinbock, A. Tóth, and K. Showalter, Science 267, 868 (1995).

[29] K. I. Agladze, R. R. Aliev, T. Yamaguchi, and K. Yoshikawa, J. Phys. Chem. 100, 13895 (1996).

[30] J. Gorecki, J. N. Gorecka, K. Yoshikawa, Y. Igarashi, and H.
Nagahara, Phys. Rev. E 72, 046201 (2005).

[31] I. Motoike and K. Yoshikawa, Phys. Rev. E 59, 5354 (1999).

[32] A. Tóth, D. Horváth, and K. Yoshikawa, Chem. Phys. Lett. 345, 471 (2001).

[33] M. A. Liauw, J. Ning, and D. Luss, J. Chem. Phys. 104, 5657 (1996).

[34] S. Shvartsman, A. K. Bangia, M. Bär, and I. G. Kevrekidis, in Mathematics of Multiscale Materials, edited by K. Golden, G. R. Grimet,R. D. James, G. W. Milton, and P. N. Sen, IMA Volumes in Mathematics and its Applications(Springer, New York, 1998), pp. 231-253, Vol. 99, pp. 231-253.

[35] K. John and M. Bär, Phys. Biol. 2, 123 (2005).

[36] J. Keener and J. Sneyd, Mathematical Physiology (SpringerVerlag, New York, 1998).

[37] Y.-X. Li, Physica D 186, 27 (2003).

[38] P. C. Bressloff, S. E. Folias, A. Prat, and Y.-X. Li, Phys. Rev. Lett. 91, 178101 (2003).

[39] J. P. Pauwelussen, Physica D 4, 67 (1981).

[40] X. Yuan, T. Teramoto, and Y. Nishiura, Phys. Rev. E 75, 036220 (2007).

[41] Y. Nishiura, T. Teramoto, and K.-I. Ueda, Phys. Rev. E 67, 056210 (2003).

[42] Y. Nishiura, T. Teramoto, and K.-I. Ueda, Chaos 13, 962 (2003).

[43] Y. Nishiura, T. Teramoto, and K.-I. Ueda, Chaos 15, 047509 (2005).

[44] T. Teramoto, K.-I. Ueda, and Y. Nishiura, Phys. Rev. E 69, 056224 (2004).

[45] Y. Nishiura, T. Teramoto, X. Yuan, and K.-I. Ueda, Chaos 17, 037104 (2007).

[46] T. Teramoto, K.-I. Ueda, X. Yuan, and Y. Nishiura, in Proceedings in Information and Communications Technology, edited by Y. Suzuki Yashuhiro M. Hagiya, H. Umeo, and A. Adamatzky (Springer, Tokyo, 2009), pp. 238-249.

[47] P. van Heijster, A. Doelman, and T. J. Kaper, Physica D 237, 3335 (2008).

[48] S.-I. Ei, J. Dyn. Differ. Equ. 14, 85 (2002).

[49] S.-I. Ei, H. Ikeda, and T. Kawana, Jpn. J. Ind. Appl. Math. 25, 117 (2008).

[50] S.-I. Ei, M. Mimura, and M. Nagayama, Physica D 165, 176 (2002).

[51] Y. Nishiura, Y. Oyama, and K.-I. Ueda, Hokkaido Math. J. 36, 207 (2007).

[52] Y. A. Kuznetsov, Elements of Applied Bifurcation Theory, 2nd ed. (Springer-Verlag, Berlin, 1998).

[53] K. Lust, D. Roose, A. Spence, and A. R. Champneys, SIAM J. Sci. Comput. (USA) 19, 1188 (1998).

[54] J. Krishnan, K. Engelborghs, M. Bär, K. Lust, D. Roose, and I. G. Kevrekidis, Physica D 154, 85 (2001).

[55] J. Guckenheimer and P. Holmes, Nonlinear Oscillations, Dynamical Systems, and Bifurcations of Vector Fields (Springer, Berlin, 1983). 